\title{
The most efficient critical vaccination coverage and its equivalence with maximizing the herd effect
}

\author{
Econometric Institute Report 2016 - 06 \\ Evelot Duijzer*, Willem van Jaarsveld’ Jacco Wallinga ${ }^{\ddagger}$, Rommert Dekker* \\ February 18, 2016
}

\begin{abstract}
'Critical vaccination coverages' are vaccination allocations that result in an effective reproduction ratio of one. In a population with interacting subpopulations there are many different critical vaccination coverages. To find the most efficient critical vaccination coverage, we define the following optimization problem: minimize the required amount of vaccines to obtain an effective reproduction ratio of exactly one. We prove that this optimization problem is equivalent to the problem of maximizing the proportion of susceptibles that escape infection during an epidemic (i.e., maximizing the herd effect).

We propose an efficient general algorithm to solve these optimization problems based on PerronFrobenius theory. We study two special cases that provide further insight into these optimization problems. First, we derive an explicit analytic solution for the case of two interacting populations. Second, we derive an efficient algorithm for the case of multiple populations that interact according to separable mixing. In this algorithm the subpopulations are ordered by their ratio of population size to reproduction ratio. Allocating vaccines based on this priority order results in an optimal allocation. We apply our solutions in a case study for pre-pandemic vaccination in the initial phase of an influenza pandemic where the entire population is susceptible to the new influenza virus. The results show that for the optimal allocation the critical vaccination coverage is achieved for a much smaller amount of vaccines as compared to allocations proposed previously.
\end{abstract}

Keywords: infectious diseases, vaccination, reproduction number, heterogeneous mixing, optimization, mathematical model

\footnotetext{
*Econometric Institute, Erasmus University Rotterdam, The Netherlands, \{duijzer, rdekker\}@ese.eur.nl

${ }^{\dagger}$ Department of Industrial Engineering and Innovation Sciences, Eindhoven University of Technology, The Netherlands, W.L.v.Jaarsveld@tue.nl

${ }^{\ddagger}$ National Institute for Public Health and The Environment (RIVM), Bilthoven, The Netherlands, jacco.wallinga@rivm.nl
} 


\section{Introduction}

In infectious disease epidemiology the potential of an infectious agent to cause an epidemic is often expressed in terms of the reproduction ratio and the final size. The final size is the eventual number of people that have become infected. The reproduction ratio, denoted by $R$, is considered to be one of the most important parameters in infectious disease epidemiology and has received considerable attention (cf. Diekmann, Heesterbeek, \& Britton, 2012). The effectiveness of a control strategy against the infectious agent is often expressed as the capability of the strategy to reduce the reproduction ratio or the final size. Several studies focus on the minimization of $R$ under a capacity constraint on the available resources (e.g. Goldstein et al., 2009; Wallinga, van Boven, \& Lipsitch, 2010) or on the threshold criterion $R=1$ (e.g. Britton, Becker, et al., 2000; Hill \& Longini Jr, 2003). $R$ is rather tractable and hence the above papers typically use analytical methods based on matrix algebra. In contrast, applying analytical methods to minimizing the final size is more difficult, as the final size is implicitly defined. Therefore, numerical evaluation (e.g. Arino, Brauer, Van Den Driessche, Watmough, \& Wu, 2008; Keeling \& Shattock, 2012; Yuan, Alderson, Stromberg, \& Carlson, 2015) or simulation (e.g. Andradóttir, Chiu, Goldsman, \& Lee, 2014; Cooper, Pitman, Edmunds, \& Gay, 2006; Ferguson et al., 2005) are typically used to analyze the final size.

There is no obvious connection between minimization of the reproduction ratio $R$ and minimization of the final size. It is not clear how an intervention that minimizes $R$ affects the final size and vice versa. Tildesley and Keeling (2009) even show that the reproduction ratio within a population is a bad predictor for the final size when populations interact. The relation between $R$ and the final size has been studied for a single population and a one-to-one relation can be derived (Ma \& Earn, 2006). However, this relation does not extend to multiple populations.

A first step in analyzing the relation between $R$ and the final size for multiple populations is made by Andreasen (2011) for the case without infection control. The initial population is then completely susceptible and the reproduction ratio $R$ equals the basic reproduction ratio $R_{0}$. Andreasen (2011) shows that an epidemic occurs only for $R_{0}>1$, implying that the final size equation has an interior solution in that case. In case $R_{0} \leq 1$ only the boundary solution exists, corresponding to no outbreak. We build upon Andreasen (2011) by including vaccination in a completely susceptible population and assuming that the disease is introduced after vaccination. In a vaccinated population the final size is determined by the direct effect of vaccination and the indirect effect. This latter effect is also known as the herd effect. The direct effect is measured as the proportion of the people that are protected from infection by vaccination, whereas the indirect herd effect is measured as the proportion of the people that are not exposed to infection and thus 
escape infection without being vaccinated. The herd effect can be influenced by the vaccine allocation and is therefore the most interesting.

We are interested in finding vaccine allocations that maximize the herd effect and we define the following optimization problem: maximize the overall herd effect. This problem is difficult to solve (Keeling \& Ross, 2015). We show that formulating the equivalent optimization problem in terms of $R$ enables to solve the problem. We show analytically that the herd effect in a set of populations can only be maximized for a vaccination allocation that results in $R=1$. In previous work we already showed that this holds for a single population (Duijzer, van Jaarsveld, Wallinga, \& Dekker, 2015), we extend this in the current paper to interacting populations. We define a second optimization problem: minimize the required amount of vaccines to obtain $R=1$. We prove that the two optimization problems are equivalent. This allows us to propose a solution method based on Perron-Frobenius Theory (Meyer, 2000). We then consider two special cases: the case of separable mixing and the case of $n=2$ populations. The case of separable mixing is often studied and assumes that upon transmission from one individual to another the two individuals involved influence transmission independently (Diekmann et al., 2012). For these two special cases we are able to characterize the optimal solution completely. The algorithm for separable mixing provides especially interesting insights: we show that vaccinating according to a very simple priority ordering based on population size and disease parameters results in the optimal allocation. We illustrate our approach to find the optimal allocation in a case study for pre-pandemic vaccination in the initial phase of an impending influenza pandemic. The results show that the amount of required vaccines to attain $R=1$ can differ substantially if we compare the optimal allocation with proposed allocations in literature.

The remainder of this paper is structured as follows. In Section 2 we formulate the problem: The herd effect and the reproduction ratio $R$ are presented and illustrated for the standard epidemiological $S I R$ model. Next, we formulate the two vaccine allocation problems that are the main focus of the paper. Section 3 discusses the assumptions and some technical details that are needed for the analysis of the optimization problems. In Section 4 we prove that the two vaccine allocation problems are equivalent. Section 5 is dedicated to solving these problems. Section 6 contains an application of our solution method. We conclude the paper with a discussion in Section 7 and conclusions in Section 8. 


\section{Problem formulation}

\subsection{The $S I R$ model}

We consider the standard epidemiological $S I R$ model for a set $J$ consisting of $n$ interacting populations indexed by $j$, i.e., $|J|=n$. Every population is divided into three compartments for which the evolution is tracked (cf. Hethcote, 2000). Let $s_{j}(t), i_{j}(t)$ and $r_{j}(t)$ be the fractions of population $j$ respectively susceptible, infected and removed at time $t$. Let $\gamma_{j}$ denote the recovery rate in population $j$ and let $\beta_{j l}$ denote the transmission rate between susceptible people from population $j$ and infected people from population $l$. The $S I R$ model describes the time course of an epidemic and consists of the following system of differential equations:

$$
\begin{aligned}
\frac{d s_{j}}{d t} & =-\sum_{l \in J} \beta_{j l} s_{j} i_{l} & & \forall j \in J \\
\frac{d i_{j}}{d t} & =\sum_{l \in J} \beta_{j l} s_{j} i_{l}-\gamma_{j} i_{j} & & \forall j \in J \\
\frac{d r_{j}}{d t} & =\gamma_{j} i_{j} & & \forall j \in J
\end{aligned}
$$

Figure 1 illustrates the time course of an epidemic according to the SIR model. As time progresses the number of infected individuals will approach zero and the epidemic will die out, i.e., $\lim _{t \rightarrow+\infty} i_{j}(t)=0$ for all $j \in J$. When the state of the system no longer changes, it is in the disease free equilibrium (DFE).

We include vaccination in the $S I R$ model at time $t=0$. We assume that all individuals are vaccinated before the start of the epidemic. That the fractions of vaccinated individuals may differ between populations. Let $f_{j}$ denote the fraction of people vaccinated in population $j$. We assume that one dose of vaccine suffices and that vaccination instantaneously leads to perfect immunity against infection. For a relaxation of the assumption of perfect vaccines we refer to Appendix D. Upon vaccination the system changes from state $\left(s_{j}(0), i_{j}(0), r_{j}(0)\right)$ to state $\left(\left(1-f_{j}\right) s_{j}(0), i_{j}(0), r_{j}(0)+s_{j}(0) f_{j}\right)$ for all $j \in J$.

We are interested in the final state of an outbreak, i.e., $\lim _{t \rightarrow+\infty} s_{j}(t)$, which depends on the initial state at time 0 . In the remainder of this paper we consider one specific initial state, namely the situation that vaccination takes place prior to an outbreak in a completely susceptible population. In literature this type of vaccination is called pre-pandemic vaccination. Let $\mathbf{s}_{\mathbf{0}}, \mathbf{i}_{\mathbf{0}}$ and $\mathbf{r}_{\mathbf{0}}$ respectively denote the vectors with initial fractions of people susceptible, infected and removed. For pre-pandemic vaccination it is assumed that $\mathbf{s}_{\mathbf{0}}=\mathbf{1}$ and $\mathbf{i}_{\mathbf{0}}=\mathbf{r}_{\mathbf{0}}=\mathbf{0}$. Note that without infected individuals, the system in (1) is in equilibrium and no transmission can occur. To analyze an outbreak after pre-pandemic vaccination many studies therefore consider that the system is externally exposed to a 'shock' or that the disease is introduced after vaccination, 


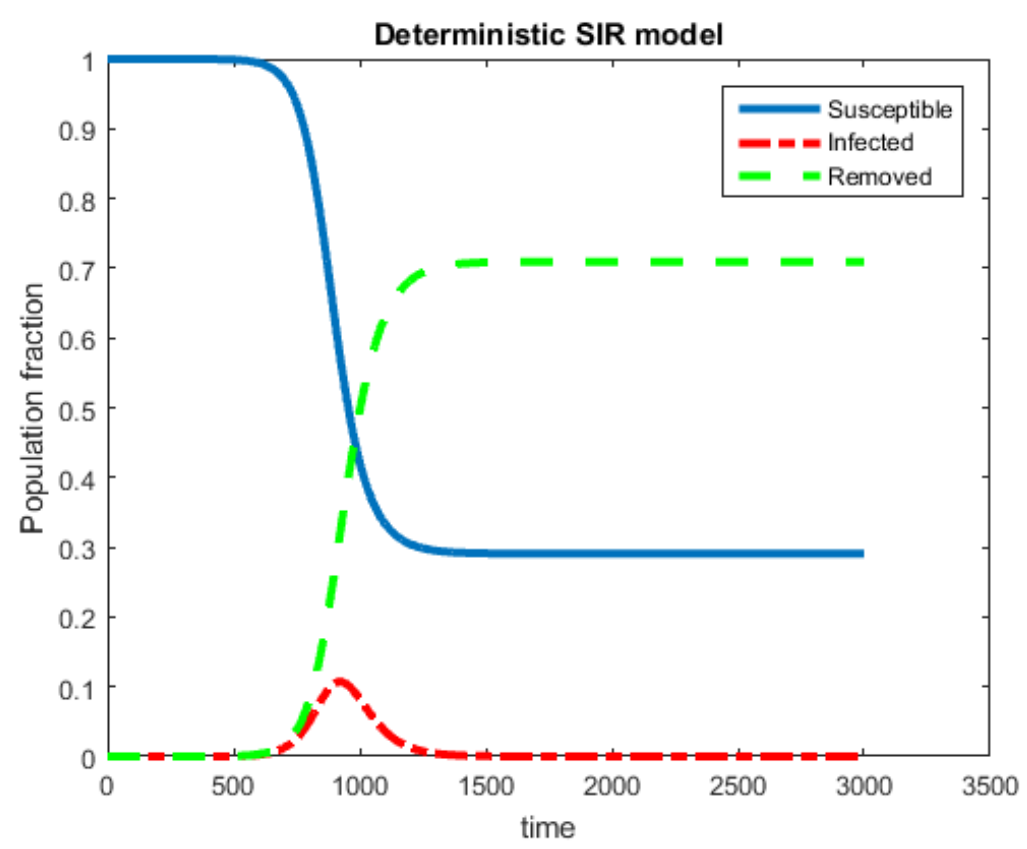

Figure 1: Illustration of the deterministic $S I R$ model for two populations with parameters $\gamma_{j}=2.3, \beta_{j j}=3$ for $j=1,2$ and $\beta_{j l}=1$ for $j \neq l$. We introduce a minor infection of $i_{j}(0)=10^{-14}$ for $j=1,2$ to analyze the time course of the epidemic. Because of symmetry between populations the time course is presented for only one population.

meaning that an infinitely small fraction of individuals gets infected. By Perron-Frobenius Theory the initial phase of an epidemic is uniquely determined (see Section 3.1) and it is therefore not necessary to specify the introduction of the disease in detail (cf. Diekmann, 1977; Metz, 1978).

\section{$2.2 \quad$ Herd effect}

Vaccination leads to people escaping infection in two ways: either directly or indirectly. The direct effect is measured as the proportion of individuals that are vaccinated themselves and hence immune. The indirect effect, also referred to as the herd effect, is measured as the proportion of individuals that are unvaccinated and escape infection because of a reduction in force of infection due to vaccination. The individuals that escape infection without being vaccinated are still susceptible in the disease free equilibrium. Denote by $G_{j}(\mathbf{f})$ the final fraction of people susceptible in population $j$, i.e., the herd effect in population $j$. Here $\mathbf{f}$ denotes the vector with the vaccination fractions $f_{j}$ for all populations $j \in J$. Then:

$$
G_{j}(\mathbf{f})=\lim _{t \rightarrow+\infty} s_{j}(t) \quad \forall j \in J
$$


Denote by $\sigma_{j}=\frac{\beta_{j j}}{\gamma_{j}}$. From (1) we derive the functions $G_{j}(\mathbf{f})$ for all $j \in J$ using the initial conditions of pre-pandemic vaccination. This leads to the following implicit set of equations for the herd effect:

$$
0=-G_{j}(\mathbf{f})+\frac{\log \left(G_{j}(\mathbf{f})\right)}{\sigma_{j}}-\frac{1}{\sigma_{j}} \log \left[\left(1-f_{j}\right)\right]+\left(1-f_{j}\right)+\frac{1}{\sigma_{j}} \sum_{l \in J: l \neq j} \frac{\beta_{j l}}{\gamma_{l}}\left[1-f_{l}-G_{l}(\mathbf{f})\right] \quad \forall j \in J
$$

For the remainder of the paper it is more convenient to reformulate (3) in matrix notation. Denote by $\gamma, \sigma$ and $\mathbf{B}$ the following matrices, with $\gamma$ and $\sigma$ diagonal matrices:

$$
\gamma=\left[\begin{array}{ccc}
\frac{1}{\gamma_{1}} & & 0 \\
& \ddots & \\
0 & & \frac{1}{\gamma_{n}}
\end{array}\right] \quad \sigma=\left[\begin{array}{ccc}
\frac{-1}{\sigma_{1}} & & 0 \\
& \ddots & \\
0 & & \frac{-1}{\sigma_{n}}
\end{array}\right] \quad \mathbf{B}=\left[\begin{array}{ccc}
\beta_{11} & \cdots & \beta_{1 n} \\
\vdots & \ddots & \vdots \\
\beta_{n 1} & \cdots & \beta_{n n}
\end{array}\right]
$$

Furthermore, let $\mathbf{G}$ denote the vector $\left[G_{1}(\mathbf{f}) \cdots G_{n}(\mathbf{f})\right]^{T}$. Let $\mathbf{1}$ denote the all ones vector of length $n$. Then (3) can be written as follows, with $\log (\cdot)$ used element wise.

$$
\sigma \log (\mathbf{G})=\sigma \log (\mathbf{1}-\mathbf{f})-\sigma \mathbf{B} \gamma[\mathbf{1}-\mathbf{f}-\mathbf{G}]
$$

We used the $S I R$ model to illustrate the implicit expression for the herd effect. This implicit expression is an extension of the final size formula, for which the generality is shown in Ma and Earn (2006). The final size of an outbreak equals the total number of people that have become infected and is therefore directly related to the number of people that have escaped infection, i.e., the total effect of vaccination. This total effect is the sum of the direct effect and the herd effect.

\subsection{Reproduction ratio}

The basic reproduction ratio, denoted by $R_{0}$, is defined as the number of new infections caused by a single infectious individual in a completely susceptible population. In the initial phase of an epidemic there are very few infected individuals, so the population is almost completely susceptible. $R_{0}$ is therefore related to the exponential initial growth rate of epidemic (cf. Wallinga \& Lipsitch, 2007). For compartmental models $R_{0}$ can be determined from the differential equations (Diekmann et al., 2012).

After vaccination the population is no longer completely susceptible, so we can no longer use the basic reproduction ratio. In this paper we therefore consider the effective reproduction ratio. To formally define the effective reproduction ratio we introduce the following notation: Let $\mathbf{S}(\mathbf{t})$ denote the diagonal matrix with entries $s_{j}(t)$, and let $\mathbf{B}$ and $\gamma$ be defined in accordance to (4).

Definition 1. The effective reproduction ratio at time $t$, denoted by $R_{e}(t)$, is determined as follows: $R_{e}(t)=\rho(\mathbf{S}(\mathbf{t}) \mathbf{B} \gamma)$, with $\rho(\cdot)$ denoting the spectral radius, i.e., the largest eigenvalue. 
The matrix $\mathbf{S}(\mathbf{t}) \mathbf{B} \gamma$ is also referred to as the next generation matrix at time $t$. Hence, the effective reproduction ratio is the largest eigenvalue of the next generation matrix. Note that the effective reproduction ratio at time $t$ does only depend on the parameters and on the fractions of individuals susceptible. The fractions of infected individuals do not play a role. We denote by $R_{f}=R_{e}(0)$ the effective reproduction ratio at time 0 , directly after vaccination. At time $t=0$ it holds that $s_{j}(0)=\left(1-f_{j}\right)$ for all $j \in J$. This implies that $R_{f}=\rho(\mathbf{F B} \gamma)$, with $\mathbf{F}$ denoting the following diagonal matrix:

$$
\mathbf{F}=\left[\begin{array}{ccc}
\left(1-f_{1}\right) & & 0 \\
& \ddots & \\
0 & & \left(1-f_{n}\right)
\end{array}\right]
$$

Vaccination allocations $\mathbf{f}$ that result in $R_{f}=1$ are referred to as 'critical vaccination fractions' or 'critical vaccination coverages'. In a homogeneous population, or in a population where vaccine is allocated at random, such a critical vaccination coverage would be a single number. Here we deal with multiple interacting populations, where vaccine is not distributed at random, and hence we have multiple critical vaccination coverages, each of which is a vector with a vaccination fraction for every population. In Section 4 critical vaccination coverages and their importance for the herd effect are discussed further.

\section{$2.4 \quad$ Vaccine allocation problems}

Using the herd effect and the basic reproduction ratio we define two vaccine allocation problems. The herd effect relates to unvaccinated individuals benefitting from the vaccination of others. An efficient allocation thus makes the best possible use of the herd effect. We define the optimization problem of maximizing the herd effect:

$$
\begin{array}{ll}
\max & \sum_{j \in J} N_{j} G_{j}(\mathbf{f}) \\
\text { s.t. } & 0 \leq \mathbf{f} \leq 1
\end{array}
$$

In words, we maximize the number of susceptible individuals at the end of the epidemic, subject to the condition that the vaccine allocation $\mathbf{f}$ consists of proper proportions between 0 and 1 . The objective is to maximize the total herd effect (7), with $N_{j}$ denoting the size of population $j$. The fact that the herd effect is implicitly defined (see (3)) significantly complicates the analysis of this optimization problem. Therefore, papers that focus on maximizing the herd effect, or relatedly on minimizing the final size under a capacity constraint, typically rely on numerical evaluation or enumeration to determine the optimal allocation (e.g. Arino et al., 2008; Keeling \& Shattock, 2012; Yuan et al., 2015). 
We also define a vaccine allocation problem based on the reproduction ratio, using the important threshold $R_{f}=1$. For this threshold an outbreak will not lead to an increase in infected individuals (cf. Section 4). There are multiple critical vaccination coverages, i.e., vaccination allocations that result in $R_{f}=1$. We are interested in finding the critical vaccination coverage that uses the least amount of vaccines, referred to as 'the most efficient critical vaccination coverage'. This leads to the following optimization problem that minimizes the required amount of vaccines such that $R_{f}=1$ :

$$
\begin{array}{ll}
\min & \sum_{j \in J} N_{j} f_{j} \\
\text { s.t. } & \rho(\mathbf{F B} \gamma)=1 \\
& 0 \leq \mathbf{f} \leq 1
\end{array}
$$

In words, we minimize the number of vaccinated individuals under the condition that the reproduction ratio is precisely one and the vaccine allocation $\mathbf{f}$ is properly defined. Constraint (10) implies $R_{f}=1$. Problem (9) (11) has been studied before in literature. Hill and Longini Jr (2003) extensively study critical vaccination coverages and also pay some attention to minimizing the number of allocated vaccines. The authors propose to solve the problem using Lagrangian multipliers, which they acknowledge to be computationally inefficient. For a stochastic epidemic spread and a population split up in households the problem can be approximated with a linear programming problem (Ball \& Lyne, 2002; Becker \& Starczak, 1997; Keeling \& Ross, 2015).

The remainder of this paper is dedicated to analyzing the two vaccine allocation problems presented in this section. We prove their equivalence and present explicit solutions for two special cases.

\section{Preliminary analysis}

In Section 3.1 we present some technical assumptions on the model and in Section 3.2 we derive a number of basic technical results. This groundwork is needed for the analysis of the optimization problems of Section 2.4.

\subsection{Assumptions}

We make the following assumption with respect to the parameters:

Assumption 2. The parameters $\gamma_{j}$ and $\beta_{j j}$ are strictly positive for all $j \in J$ and $\beta_{i j}$ are nonnegative for all $i, j \in J$ with $i \neq j$. 
Assumption 3. The matrix $\mathbf{B}$ with elements $\beta_{i j}$ is irreducible.

The recovery rates $\gamma_{j}$ are assumed to be strictly positive. If $\gamma_{j}$ would equal 0 for some population $j$ an infected individual in population $j$ would remain infectious forever, which is unrealistic. The parameters $\beta_{i j}$ represent the transmission rates between populations $i$ and $j$. It is reasonable to assume that these rates are nonnegative. Note that $\beta_{i j}=0$ implies that there is no transmission between population $i$ and $j$. This is for example the case when there is no direct contact between the two populations.

By Assumption 2 and 3 the matrix $\mathbf{B}$ and the product $\mathbf{B} \gamma$ are nonnegative and irreducible which allows us to use Perron-Frobenius Theory (Meyer, 2000). The irreducibility of the matrix ensures that all populations interact with each other. This interaction is either direct or indirect, i.e., via other populations. Excluding the unlikely possibility that the disease can be transmitted from population $i$ to population $j$ but not vice versa, the assumption that the matrix $\mathbf{B}$ is irreducible does not restrict generality. Namely, if $\mathbf{B}$ would be reducible, the problem can be decomposed into subproblems each consisting of a subset of populations with an irreducible transmission matrix.

By Perron-Frobenius Theory a nonnegative and irreducible matrix has a unique positive eigenvector corresponding to the largest eigenvalue. This eigenvector represents the initial distribution of infected individuals over the populations and uniquely determines the initial phase of the epidemic.

\subsection{Basic technical results}

In this section we derive a number of technical results to formally proof our equivalence results in Section 4. In Lemma 15 in Appendix A we formally prove that the differential equations (1) behave in accordance to interpretation: $s_{j}(t), i_{j}(t), r_{j}(t) \in[0,1]$ and $s_{j}(t)+i_{j}(t)+r_{j}(t)=1$ for all $j \in J$ and $t \geq 0$. Furthermore, the fraction of susceptible people is non-increasing over time and the fraction of removed people is non-decreasing over time. The next theorem formally shows that the differential equations of the $S I R$ model have a unique solution.

Theorem 4. Given the initial values $s_{j}(0), i_{j}(0), r_{j}(0) \in[0,1]$ such that $s_{j}(0)+i_{j}(0)+r_{j}(0)=1$ for all $j \in J$ the differential equations in (1) have a unique solution at any time $t$.

Proof. We prove that the differential equations in (1) are Lipschitz continuous (see Appendix A). By the Picard-Lindelöf Theorem (Lindelöf, 1894) there is a unique solution to the initial value problem.

The following lemma establishes bounds on the herd effect $G_{j}(\mathbf{f})$, that is characterized in (3). We use these bounds in later sections. 
Lemma 5. For all $j \in J$ the following holds:

(i) $0 \leq G_{j}(\mathbf{f}) \leq \min \left\{\left(1-f_{j}\right), \frac{1}{\sigma_{j}}\right\}$

(ii) $G_{j}(\mathbf{f})=0$ if and only if $f_{j}=1$.

Proof. See Appendix A.

\section{Equivalence results}

We are now able to analyze in detail the relation between the basic reproduction ratio and the herd effect. Section 4.1 relates $R_{f}=\rho(\mathbf{F B} \gamma)$ to the solutions to the implicit expression of the herd effect in equation (5). Based on this relation we prove in Section 4.2 that the two optimization problems of Section 2.4 are equivalent.

\subsection{The relation between $R_{f}$ and the herd effect}

The stability of disease free equilibria and the relation with $R_{0}$ has been investigated for different types of compartmental models (among others Andreasen, 2011; Hu, Teng, \& Jiang, 2012; Van den Driessche \& Watmough, 2002). Typically, the conclusion is that a disease free equilibrium (DFE) is stable for $R_{0}<1$ and unstable for $R_{0}>1$. A DFE represents a solution to the final size equation, which is directly related to the herd effect as discussed before. In this section we derive the relation between $R_{f}$ and the herd effect extending known results to pre-pandemic vaccination. The pre-pandemic vaccination case can directly be translated to models without vaccination, by changing the initial fraction of people susceptible $s_{j}(0):=s_{j}(0)\left(1-f_{j}\right)$. Our definition of $R_{f}$ is directly related to $R_{0}$. We confirm the critical role of $R_{f}$ and the threshold $R_{f}=1$.

The herd effect is defined according to the implicit set of equations (5). By Lemma 5 we know that any solution $G_{j}(\mathbf{f})$ lies in the interval $\left[0,\left(1-f_{j}\right)\right]$ for all $j \in J$. By Theorem 4 the differential equations (1) have a unique solution at any point in time. This implies that there is also a unique solution for $G_{j}(\mathbf{f})$, which is a stable disease free equilibrium (DFE). Let $\tilde{\mathbf{G}}$ denote the vector with elements $G_{j}(\mathbf{f}) /\left(1-f_{j}\right)$. We use $\log (\cdot)$ element wise and rewrite (5) into:

$$
0=\log (\tilde{\mathbf{G}})+B \gamma \mathbf{F}(1-\tilde{\mathbf{G}})
$$

It can easily be verified that equation (12) always has the solution $\tilde{\mathbf{G}}=\mathbf{1}$, i.e., $G_{j}(\mathbf{f})=\left(1-f_{j}\right)$. This solution will be referred to as the trivial solution in accordance to Andreasen (2011) and corresponds to the situation of no outbreak. Recall that directly after vaccination the fraction of people susceptible equals $\left(1-f_{j}\right)$ in population $j$. Hence, in the trivial solution all susceptible people will remain susceptible. Additionally, we 
consider solutions that do correspond to outbreaks. We use the term 'interior solution' to refer to a solution for which $G_{j}(\mathbf{f}) \in\left(0,\left(1-f_{j}\right)\right)$ for all $j \in J$. We extend Lemma 1 of Andreasen (2011) to include vaccination and the case that $R_{f}$ equals 1 .

Lemma 6. For $R_{f} \leq 1$ equation (12) does not have an interior solution.

Proof. By contradiction assume that there is an interior solution, denoted by $\mathbf{Y}$. Let $\tilde{\mathbf{Y}}$ denote the vector with elements $Y_{j} /\left(1-f_{j}\right)$ which are all in $(0,1)$. Recall that $R_{f}=\rho(\mathbf{F} B \gamma)$ and let $v$ be the left eigenvector corresponding to this largest eigenvalue. From Perron-Frobenius Theory (Meyer, 2000) we know that we can choose $v$ such that all elements are nonnegative and $\|v\|_{1}=1$. Left multiplication of (12) with $v^{T} \mathbf{F}$ results in the following:

$$
\begin{aligned}
0 & =v^{T} \mathbf{F} \log (\tilde{\mathbf{Y}})+v^{T} \mathbf{F} B \gamma \mathbf{F}(1-\tilde{\mathbf{Y}}) \\
& =v^{T} \mathbf{F} \log (\tilde{\mathbf{Y}})+R_{f} v^{T} \mathbf{F}(1-\tilde{\mathbf{Y}}) \\
& =v^{T} \mathbf{F}[\log (\tilde{\mathbf{Y}})+(1-\tilde{\mathbf{Y}})]+\left(R_{f}-1\right) v^{T} \mathbf{F}(1-\tilde{\mathbf{Y}})
\end{aligned}
$$

Note that $v^{T} \mathbf{F} \in(0,1)^{n}$ and also $(1-\tilde{\mathbf{Y}}) \in(0,1)^{n}$. Furthermore, using that $\log x<x-1$ for $x \neq 1$ we derive:

$$
[\log (\tilde{\mathbf{Y}})+(1-\tilde{\mathbf{Y}})]<0
$$

Thus, the third line of (13) is the summation of a strictly negative and a nonpositive term for $R_{f} \leq 1$ and is therefore strictly negative. We arrive at a contradiction, which completes the proof of this lemma.

The interpretation of Lemma 6 is as follows: in case $R_{f} \leq 1$ the system is in a stable disease free equilibrium. Introduction of a disease in the population will not lead to an outbreak in that case.

To analyze the case that $R_{f}>1$ we use a variable transformation and introduce the variable vector $\mathbf{x}(\mathbf{f})$ with elements $x_{j}(\mathbf{f})=1-\frac{G_{j}(\mathbf{f})}{1-f_{j}}$. We rewrite (5) into:

$$
\mathbf{x}(\mathbf{f})=1-\exp \left\{-[\mathbf{B} \gamma \mathbf{F} \mathbf{x}(\mathbf{f})]_{j}\right\}
$$

From (14) and Lemma 5 we can derive that the variables $x_{j}(\mathbf{f})$ lie in the interval $[0,1)$ for all $j \in J$.

Theorem 7. For $R_{0}^{f}>1$ equation (12) has a unique interior solution.

Proof. This theorem is equivalent to the statement that (14) has a unique positive solution in case $R_{f}>1$. Equation (14) has a positive solution if and only if $\rho(\mathbf{B} \gamma \mathbf{F})>1$ (Theorem 3.1 of Chan, Nishiura, Diekmann, \& Bootsma, 2014). Note that $\mathbf{F}$ is an invertible matrix, which implies that the matrices $\mathbf{B} \gamma \mathbf{F}$ and $\mathbf{F B} \gamma$ are 
similar and have the same eigenvalues. Hence, $R_{f}:=\rho(\mathbf{F B} \gamma)=\rho(\mathbf{B} \gamma \mathbf{F})$. This completes the proof of this theorem.

Recall that the herd effect expression is directly related to the final size equation. Theorem 7 therefore coincides with Diekmann and Heesterbeek (2000) who claim (in exercise 6.19) that the final size equation has a unique non-trivial (i.e., non-zero) solution in case $R_{0}>1$.

\subsection{Equivalence of the two problems}

Based on the results in the previous section we prove the equivalence of the two vaccine allocation problems discussed before.

Theorem 8. The overall herd effect, $\sum_{j \in J} N_{j} G_{j}(\mathbf{f})$, is maximized for a vaccine allocation $\mathbf{f}$ that results in $R_{f}=1$.

Proof. We will prove this lemma by contradiction in two steps. First we show that the overall herd effect cannot be maximized for a vaccine allocation that results in $R_{f}<1$ and in the second step we will exclude the possibility that an allocation with $R_{f}>1$ maximizes the overall herd effect.

Step 1: by contradiction assume that there is a vaccine allocation $\mathbf{x}$ resulting in $R_{f}<1$, which maximizes the overall herd effect. Denote by $\mathbf{X}$ the diagonal matrix with entries $\left(1-x_{j}\right)$. Furthermore, define the function $g(t)=\rho[(t \mathbf{I}+(1-t) \mathbf{X}) \mathbf{B} \gamma]$ for $t \in[0,1]$. By construction $g(0)=\rho(\mathbf{X B} \gamma)<1$ and to avoid triviality we can assume that $g(1)=\rho(\mathbf{B} \gamma)>1$. By Lemma 16, which is formulated and proven in Appendix B, the function $g(t)$ is continuous in $t$. Hence, there exists a $t^{*} \in(0,1)$ for which $g\left(t^{*}\right)=1$ by the intermediate value theorem. Let $\mathbf{Y}:=t^{*} \mathbf{I}+\left(1-t^{*}\right) \mathbf{X}$. Note that $\mathbf{Y}$ is also a diagonal matrix and let the vector $\mathbf{y}$ be such that $\left(1-y_{j}\right)$ for all $j \in J$ are the diagonal elements of $\mathbf{Y}$. We compare the vaccine allocation vectors $\mathbf{y}$ and $\mathbf{x}$ : It holds that $y_{i} \leq x_{i}$ for all $i \in J$ and a strict inequality for at least one population by the fact that $t^{*} \in(0,1)$. By Lemma 6 the trivial solution holds for both $\mathbf{x}$ and $\mathbf{y}$, because $\rho(\mathbf{X B} \gamma)<1$ and $\rho(\mathbf{Y B} \gamma)=1$. Hence,

$$
\sum_{j \in J} N_{j} G_{j}(\mathbf{y})=\sum_{j \in J} N_{j}\left(1-y_{j}\right)>\sum_{j \in J} N_{j}\left(1-x_{j}\right)=\sum_{j \in J} N_{j} G_{j}(\mathbf{x})
$$

We arrive at a contradiction: $\mathbf{x}$ cannot maximize the overall herd effect. Thus, we conclude that the overall herd effect is maximized for a vaccination fraction that results in $R_{f} \geq 1$.

Step 2: by contradiction assume that there is a vaccine allocation $\mathbf{z}$ resulting in $R_{f}>1$, which maximizes the overall herd effect. By definition of $R_{f}$ we have that $R_{e}(0)>1$ which implies by Lemma 21 
(see Appendix B) that $\lim _{t \rightarrow+\infty} R_{e}(t)<1$. We know that $R_{e}(t)$ is continuous in $t$ (Lemma 19 in Appendix B) and hence by the intermediate value theorem there exists a time $\tau>0$ at which $R_{e}(\tau)=1$. Since $\lim _{t \rightarrow+\infty} R_{e}(t)<R_{e}(\tau)$ and $R_{e}(t)$ can only decrease by a decrease in the fraction of people susceptible, the following holds:

$$
\sum_{j \in J} N_{j} G_{j}(\mathbf{z})=\lim _{t \rightarrow+\infty} \sum_{j \in J} N_{j} s_{j}(t)<\sum_{j \in J} N_{j} s_{j}(\tau)
$$

Let us now consider an alternative vaccination allocation denoted by $\mathbf{y}$, such that $y_{j}=\left(1-s_{j}(\tau)\right)$ for all $j \in J$. The definition of $R_{e}(\tau)$ does not depend on the fraction of people infected at time $\tau$. By construction we thus have that $\mathbf{y}$ results in $R_{f}=1$. From Lemma 6 we conclude the following:

$$
\sum_{j \in J} N_{j} G_{j}(\mathbf{y})=\sum_{j \in J} N_{j}\left(1-y_{j}\right)=\sum_{j \in J} N_{j} s_{j}(\tau)>\sum_{j \in J} N_{j} G_{j}(\mathbf{z})
$$

where the inequality follows from (15). We arrive at a contradiction: $\mathbf{z}$ cannot maximize the overall herd effect.

We conclude that the vaccine allocation $\mathbf{f}$ that maximizes the overall herd effect cannot result in $R_{f}>1$ nor in $R_{f}<1$. Thus, $R_{f}$ must equal 1 , which completes the proof of this lemma.

Theorem 9 presents the main result of this section:

Theorem 9. Problem (7) - (8) and Problem (9) - (11) are equivalent.

Proof. We conclude the following:

$$
\begin{array}{|cc}
\max & \sum_{j \in J} N_{j} G_{j}(\mathbf{f}) \\
\text { s.t. } & \mathbf{f} \in[0,1]^{n}
\end{array} \Leftrightarrow \Leftrightarrow \begin{array}{cc}
\max & \sum_{j \in J} N_{j} G_{j}(\mathbf{f}) \\
\text { s.t. } & R_{f}=1 \\
& \mathbf{f} \in[0,1]^{n}
\end{array} \Leftrightarrow\left[\begin{array} { c c } 
{ \operatorname { m a x } } & { \sum _ { j \in J } N _ { j } ( 1 - f _ { j } ) } \\
{ \text { s.t. } } & { R _ { f } = 1 } \\
{ } & { \mathbf { f } \in [ 0 , 1 ] ^ { n } }
\end{array} \Leftrightarrow \left[\begin{array}{ll}
\min & \sum_{j \in J} N_{j} f_{j} \\
\text { s.t. } & R_{f}=1 \\
\mathbf{f} \in[0,1]^{n}
\end{array}\right.\right.
$$

The first implication follows from Theorem 8. For the second implication we apply Lemma 6 which states that $G_{j}(\mathbf{f})=\left(1-f_{j}\right)$ for all $j \in J$ in case $R_{f}=1$.

By Theorem 9 the two optimization problems presented in Section 2.4 are equivalent. The intuitive explanation of this result is that the problem of minimizing the number of vaccines to attain $R_{f}=1$ requires a very efficient allocation of vaccines. Maximizing the herd effect thus leads to the most efficient allocation of vaccines. 


\section{Solving the problems}

Optimization problem (9) - (11) has a simple linear objective function. The main difficulty of this problem is the constraint $R_{f}=1$, which is not in general convex (or concave) (c.f. Hill \& Longini Jr, 2003). By Theorem 9 Problem (7) - (8) can also be formulated as an optimization problem with a linear objective function and the constraint $R_{1}=1$. In this section we show that this constraint simplifies for two special cases. For these cases the vaccine allocation problem can be solved to optimality. In Section 5.1 we assume a special structure on the contact matrix B: separable mixing. Section 5.2 studies the important special case of two populations. Finally, in Section 5.3 a solution method is presented that is able to solve the vaccine allocation problems for the general case, without additional assumptions on the contact matrices or the number of populations.

\subsection{Separable mixing}

For notational convenience we will use $k_{i j}$ to denote the elements of the matrix $\mathbf{B} \gamma$. We consider a special structure on the matrix $\mathbf{B} \gamma$, where $k_{i j}=a_{i} b_{j}$. This structure is called separable mixing and means that population $j$ is equally susceptible to all other populations and population $i$ is equally infectious to all other populations (Diekmann et al., 2012). The special case that $a$ is proportional to $b$, i.e., $a_{i}=\delta b_{i}$ for all $i \in J$, is called proportionate mixing. Separable and proportionate mixing are often studied (e.g., Cairns, 1989; Hethcote \& Van Ark, 1987; Ross \& Black, 2014). For separable mixing $R_{f}$ can explicitly be determined.

Denote by $\operatorname{Tr}(\cdot)$ the trace of a matrix and let $\sigma_{j}=\frac{\beta_{j j}}{\gamma_{j}}$. By definition $\sigma_{j}$ can be seen as an internal reproduction ratio in population $j$. For separable mixing $R_{f}$ is defined as follows:

$$
R_{f}=\operatorname{Tr}(\mathbf{F B} \gamma)=\sum_{j \in J} \frac{\beta_{j j}}{\gamma_{j}}\left(1-f_{j}\right)=\sum_{j \in J} \sigma_{j}\left(1-f_{j}\right)
$$

By Lemma 6 and Lemma 18 any solution for which $R_{f}<1$ can never be optimal. Hence, the solution to Problem 9 - (11) does not change if we relax the constraint $R_{f}=1$ to $R_{f} \leq 1$. The linear definition of $R_{f}$ for separable mixing significantly simplifies the optimization problems of Section 2.4 which now become:

$$
\begin{array}{ll}
\max & \sum_{j \in J} N_{j}\left(1-f_{j}\right) \\
\text { s.t. } & \sum_{j \in J} \sigma_{j}\left(1-f_{j}\right) \leq 1 \\
& 0 \leq \mathbf{f} \leq 1
\end{array}
$$


In words, we maximize the herd effect subject to the constraint that $R_{f} \leq 1$ and properly defined vaccination fractions.

Based on the relaxation we are able to derive a solution method for the problem. Observe that Problem (16) - (18) is the linear programming (LP) relaxation of a knapsack problem, one of the basic problems in combinatorial optimization. This LP relaxation can be solved to optimality by a simple greedy algorithm (Dantzig, 1957). A greedy algorithm is a procedure that consecutively makes decisions that are locally optimal. For the LP relaxation of a knapsack problem such a procedure also results in the global optimum. We propose the greedy algorithm in Algorithm 1 to solve Problem (16) - (18) to optimality.

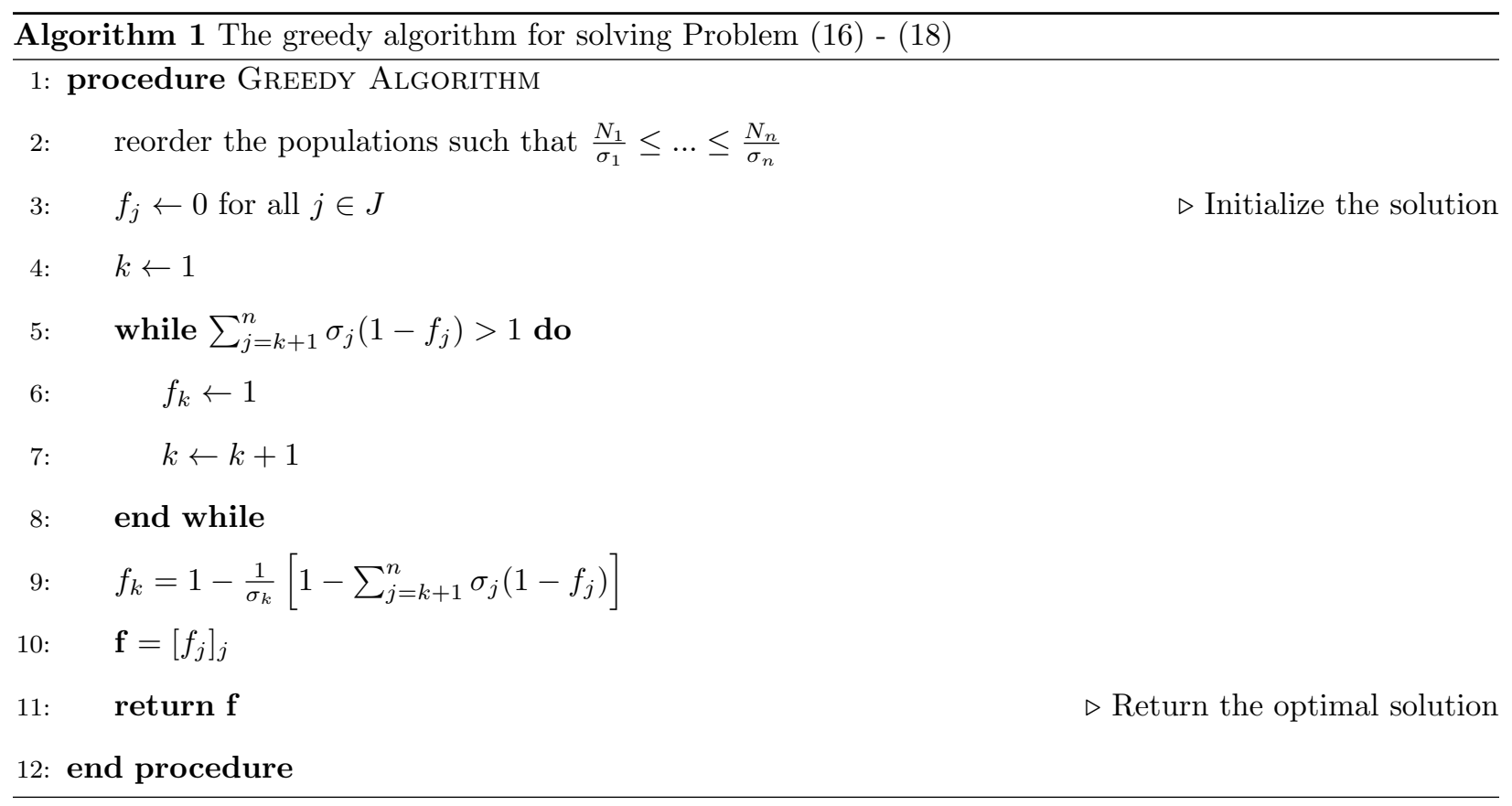

The ordering of the populations in line 2 can be done without loss of generality. The allocation resulting from Algorithm 1 prioritizes small populations and populations with a high $\sigma_{j}$. Vaccinating these populations costs relatively few vaccines, but has a large impact on lowering the reproduction ratio $R_{f}$. Note that population $j$ has a high $\sigma_{j}$ either because this population has a high transmission rate or a long infectious period (and therefore a low recovery rate). i.e., either $\beta_{j j}$ is high or $\gamma_{j}$ is low. Because of the structure of separable mixing, a population with a high internal transmission rate also plays an important role in the transmission between itself and other populations. Thus, the prioritized populations either contribute heavily to the transmission or have a long infectious period. The algorithm uses these simple characteristics to derive the vaccination allocation that results in the highest overall herd effect (and equivalently, the most efficient critical vaccination coverage). 
The optimal order in the greedy algorithm is identical to the optimal order reported for a related problem studied by Cairns (1989), who considers vaccination with a fixed rate during a time interval and the objective of minimizing the effective reproduction ratio at a certain time. The authors prove that for separable mixing it is optimal to allocate all vaccination effort to one population during a specific time interval. During consecutive time intervals the populations are vaccinated in order of increasing activity, which is exactly the order used in our greedy algorithm. Although the problem studied by Cairns (1989) differs in many respects from the problem in this paper, it is interesting to observe that under the assumptions of separable mixing the same optimal population ordering is found.

\subsection{Two populations}

For two populations an explicit expression for the condition $R_{f}=1$ is derived in Section 5.2.1. Based on this expression we are able solve the optimization problem explicitly. In Section 5.2.2 we present this explicit solution.

\subsubsection{Explicit expression for $R_{f}=1$}

Recall that $R_{f}=\rho(\mathbf{F} B \gamma)$. Denote by $\sigma_{1}=\frac{\beta_{11}}{\gamma_{1}}$, by $\sigma_{2}=\frac{\beta_{22}}{\gamma_{2}}$. Denote by $d=\operatorname{det}(B \gamma)=\frac{\beta_{11} \beta_{22}-\beta_{12} \beta_{21}}{\gamma_{1} \gamma_{2}}$. We use $\operatorname{det}(\cdot)$ and $\operatorname{Tr}(\cdot)$ to denote respectively the determinant and trace of a matrix. From the definition of the largest eigenvalue for a $2 \times 2$ matrix we derive that any vaccination allocation $f_{1}, f_{2}$ for which $R_{f}=1$ satisfies the following condition:

$$
\left(1-f_{1}\right)\left(1-f_{2}\right) \operatorname{det}(\mathbf{B} \gamma)-\operatorname{Tr}(\mathbf{B} \gamma)+\sigma_{1} f_{1}+\sigma_{2} f_{2}+1=0
$$

Furthermore, we use that $\operatorname{Tr}(\mathbf{F B} \gamma)=\operatorname{Tr}(\mathbf{B} \gamma)-\sigma_{1} f_{1}-\sigma_{2} f_{2}$ and $\operatorname{det}(\mathbf{F B} \gamma)=\left(1-f_{1}\right)\left(1-f_{2}\right) \operatorname{det}(\mathbf{B} \gamma)$. After substituting $\operatorname{Tr}(\mathbf{B} \gamma)=\sigma_{1}+\sigma_{2}$ and $\operatorname{det}(\mathbf{B} \gamma)=d$ we obtain that the critical vaccination fractions satisfy the following equation:

$$
\left(1-f_{1}\right)\left(1-f_{2}\right) d-\sigma_{1}\left(1-f_{1}\right)-\sigma_{2}\left(1-f_{2}\right)+1=0
$$

Not all solutions to (19) are critical vaccination coverages. In fact, (19) is a hyperbola and all critical vaccination coverages lie on one branch of the hyperbola (cf. Hill \& Longini Jr, 2003). The part of the correct branch of the hyperbola that contains the critical vaccination coverages is characterized in the following Lemma:

Lemma 10. Let $D$ denote the feasible region for the vaccination fractions: $D=\left\{\left(f_{1}, f_{1}\right) \mid f_{1}, f_{1} \in[0,1]\right\}$. The part of the hyperbola for which $R_{f}=1$ is described by $\left(f_{1}, f_{2}\right) \in D$ and the condition $\sigma_{2}-\left(1-f_{1}\right) d>0$ 
(equivalently the very same part of the hyperbola may also be characterized by $\left(f_{1}, f_{2}\right) \in D$ and the condition $\left.\sigma_{1}-\left(1-f_{2}\right) d>0\right)$.

Proof. We first show that the condition $\sigma_{2}-\left(1-f_{1}\right) d>0$ results in the correct branch of the hyperbola. The condition $\left(f_{1}, f_{2}\right) \in D$ stipulates that the vaccination fractions are properly defined. Secondly, we show that the other condition can be derived analogously. Rewriting (19) gives:

$$
\left(1-f_{2}\right)=\frac{1-\sigma_{1}\left(1-f_{1}\right)}{\sigma_{2}-\left(1-f_{1}\right) d}
$$

From (20) we derive that the hyperbola has an asymptote for $\sigma_{2}-\left(1-f_{1}\right) d=0$. Distinguish between the following two cases: (i) $d>0$ and (ii) $d \leq 0$. First we analyze case (i): For $R_{f}=1$ we have $G_{j}(\mathbf{f})=\left(1-f_{j}\right)$ and by Lemma 5 we know that $G_{j}(\mathbf{f})<\frac{1}{\sigma_{j}}$. This implies the following:

$$
\left(1-f_{1}\right)<\frac{1}{\sigma_{1}}=\frac{\gamma_{1}}{\beta_{11}} \leq \frac{\gamma_{1} \beta_{22}}{\beta_{11} \beta_{22}-\beta_{12} \beta_{21}}=\frac{\sigma_{2}}{d}
$$

Thus, vaccination fractions that result in $R_{f}=1$ can only occur in the branch of the hyperbola for which $\sigma_{2}-\left(1-f_{1}\right) d>0$. Consider case (ii): For $d \leq 0$ the asymptote lies outside the feasible region and therefore it is not needed to specify a single branch. However, the condition $\sigma_{2}-\left(1-f_{1}\right) d>0$ is always satisfied for $\left(f_{1}, f_{2}\right) \in D$. It can thus be added without changing the solution space.

In the same way the condition on $f_{2}$ can be derived by noting that (20) can be rewritten as follows:

$$
\left(1-f_{1}\right)=\frac{1-\sigma_{2}\left(1-f_{2}\right)}{\sigma_{1}-\left(1-f_{2}\right) d}
$$

Using the same argument, we can show that the correct branch of the hyperbola is described by $\sigma_{1}-\left(1-f_{2}\right) d>$ 0 . Hence, the symmetry between the two populations is still retained.

\subsubsection{Solution}

The conditions derived in Lemma 10 to specify the part of the hyperbola that contains the critical vaccination coverages can replace the constraint $R_{f}=1$ in the vaccine allocation problem:

$$
\begin{array}{lll}
\max & N_{1}\left(1-f_{1}\right)+N_{2}\left(1-f_{2}\right) & \\
\text { s.t. } & \left(1-f_{1}\right)\left(1-f_{2}\right) d-\sigma_{1}\left(1-f_{1}\right)-\sigma_{2}\left(1-f_{2}\right)+1=0 & \\
& \sigma_{2}-\left(1-f_{1}\right) d>0 & \\
& f_{j} \in[0,1] & j=1,2
\end{array}
$$

Constraints (22) and (23) stipulate that $R_{f}=1$. It suffices to use only one of the equivalent conditions of Lemma 10. Problem (21) - (24) can be solved by substituting $\left(1-f_{2}\right)$ in the objective function using 
equality constraint (22). By setting the derivative of the objective function with respect to $f_{1}$ equal to zero we obtain a solution, which is presented in Theorem 12. However, it is possible that this solution does not satisfy (23) - (24). In that case, we will show that the optimal solution to (21) - (24) must be a boundary solution: i.e., a solution $\left(f_{1}, f_{2}\right)$ that satisfies (22) - (23) for which $f_{1} \in\{0,1\}$ and/or $f_{2} \in\{0,1\}$. It will therefore be useful to obtain an exhaustive list of all possible boundary solutions:

Lemma 11. There are always precisely two boundary solutions to Problem (21) - (24). The first solution depends on $\sigma_{1}$ :
$f_{1}=0$
$f_{2}=1-\frac{1-\sigma_{1}}{\sigma_{2}-d}$
if $\sigma_{1} \leq 1$
(ii)
$f_{1}=1-\frac{1}{\sigma_{1}}$
$f_{2}=1$
if $\sigma_{1}>1$

The second solution depends on $\sigma_{2}$ :

\begin{tabular}{|c|c|c|c|}
\hline (iii) & $f_{1}=1-\frac{1-\sigma_{2}}{\sigma_{1}-d}$ & $f_{2}=0$ & if $\sigma_{2} \leq 1$ \\
\hline$(i v)$ & $f_{1}=1$ & $f_{2}=1-\frac{1}{\sigma_{2}}$ & if $\sigma_{2}>$ \\
\hline
\end{tabular}

Proof. To derive the four boundary solutions we use that $f_{j} \in[0,1]$ for $j=1,2$ by (24). We fix the vaccination fractions per population to the two boundary values 0 and 1 . Condition (22) is used to derive the expression for the other vaccination fraction. In the remainder of the proof we analyze the feasibility of the boundary solutions with respect to constraints (23) - (24) for the different ranges of $\sigma_{1}$ and $\sigma_{2}$.

Note that solutions $(i)$ and $(i i)$ are identical in case $\sigma_{1}=1$. The same holds for solutions (iii) and (iv) in case $\sigma_{2}=1$. To investigate the feasibility of solution $(i)$ we substitute the value $f_{1}=0$ in constraint (23) and obtain that $\sigma_{2}-d>0$. This implies that solution (i) cannot satisfy constraints (23) and (24) at the same time if $\sigma_{1}>1$. Namely, in that case satisfying constraint (23) would result in $f_{2}>1$. Equivalently, solution (iii) cannot satisfy both constraints (23) and (24) if $\sigma_{2}>1$. Using constraint (24) we can easily verify that solution $(i i)$ is not feasible in case $\sigma_{1}<1$, because it would result in $f_{1}<0$. Equivalently, $\sigma_{2}<1$ renders solution (iv) infeasible.

Based on these conclusions we proved which of the boundary solutions are feasible for which values for $\sigma_{1}$ and $\sigma_{2}$. This completes the proof of this lemma.

Theorem 12. The optimal solution to Problem (21) - (24) can be found among the boundary solutions given 
in Lemma 11 and the following solution:

$$
f_{1}=1-\frac{1}{d}\left[\sigma_{2}-\sqrt{\frac{N_{2}\left(\sigma_{1} \sigma_{2}-d\right)}{N_{1}}}\right] \quad \text { and } \quad f_{2}=1-\frac{1}{d}\left[\sigma_{1}-\sqrt{\frac{N_{1}\left(\sigma_{1} \sigma_{2}-d\right)}{N_{2}}}\right]
$$

For $d \leq 0$ only the boundary solutions need to be considered.

The proof of Theorem 12 can be found in Appendix E.

\subsection{The general case}

In this section we present a general solution approach for the vaccine allocation problems. Thereto we reformulate the optimization problems using Perron-Frobenius theory (Meyer, 2000). $R_{f}$ is the largest eigenvalue of the nonnegative and irreducible matrix $\mathbf{F B} \gamma$ by Assumption 2 and 3.

Perron-Frobenius theory states that a nonnegative and irreducible matrix has exactly one right eigenvector, the so-called Perron vector which is normalized and strictly positive. This eigenvector corresponds to the largest eigenvalue and has the following epidemiological interpretation: the Perron vector is the frequency distribution over the populations of the number of cases in the initial phase of an epidemic. The Perron vector can be used to reformulate the optimization problem of Section 2.4 (cf. Hill \& Longini Jr, 2003). Let $\mathbf{v}$ denote the right eigenvector that corresponds to $R_{f}=1$. The following holds:

$$
\mathbf{F B} \gamma \mathbf{v}=\mathbf{v} \Rightarrow \sum_{j \in J}\left(1-f_{i}\right) \frac{\beta_{i j}}{\gamma_{j}} v_{j}=v_{i}
$$

We normalize the vector $\mathbf{v}$ such that $\|\mathbf{v}\|=1$, using the $\ell_{1}$-norm. The optimization problems of Section 2.4 are then equivalent to the following problem:

$$
\begin{aligned}
& \max \sum_{j \in J} N_{j}\left(1-f_{j}\right) \\
& \text { s.t. } \quad\left(1-f_{i}\right) \sum_{j \in J} \frac{\beta_{i j}}{\gamma_{j}} v_{j} \quad=v_{i} \quad i \in J \\
& \sum_{j \in J} v_{j} \quad=1 \\
& v_{j} \quad>0 \quad j \in J \\
& \left(1-f_{j}\right) \quad \in[0,1] \quad j \in J
\end{aligned}
$$

Given the objective function and the nonnegativity of the parameters $\beta_{i j}$ and $\gamma_{j}$, constraints (26) can be relaxed to:

$$
\left(1-f_{i}\right) \sum_{j \in J} \frac{\beta_{i j}}{\gamma_{j}} v_{j} \leq v_{i} \quad i \in J
$$


Lemma 13. For any solution to Problem (25) - (29) we have $f_{i} \geq 1-\frac{\gamma_{i}}{\beta_{i i}}$.

By constraints $(29)$ we have that $f_{j} \in[0,1]$. In case $\frac{\beta_{i i}}{\gamma_{i}}>1$ the lower bound derived in Lemma 13 is stronger than the bound $f_{i} \geq 0$. For $\frac{\beta_{i i}}{\gamma_{i}} \leq 1$ the lower bound of Lemma 13 is already satisfied by the nonnegativity of $f_{i}$.

Lemma 14. The feasible region of Problem (25) - (29) is not convex.

Problem (25) - (29) contains two classes of variables: $\left(1-f_{j}\right)$ and $v_{j}$ for all $j \in J$. The objective function (25) and the constraints (27) - (29) are all linear in these variables. Constraints (26) are quadratic constraint in the two classes of variables, which makes this problem a quadratically constrained programming problem (QCP). The quadratic constraints (26) are not convex.

The lack of convexity makes it difficult to solve Problem (25) - (29) to optimality. We therefore propose a solution algorithm that cannot guarantee global optimality, but works well in our numerical experiments. We implement the formulation of our solution algorithm in Matlab and use the built-in function fmincon. This function is able to minimize non-linear programming problems with different types of constraints: linear (in)equality constraints, bounds on the variables and non-linear (in)equality constraints. It is therefore suitable to solve the QCP formulation (25) - (29). The problem formulation can easily be transformed into a minimization problem by multiplying the objective function with -1 . The solution approach of fmincon is based on interior point methods and barrier functions (c.f. Waltz, Morales, Nocedal, \& Orban, 2006). Global optimality cannot be guaranteed, because constraints (26) are not convex. To reduce the likelihood of ending up in a local optimum, we propose to use a multi start approach where we solve the problem multiple times for random start solutions. In our numerical experiments we compare the outcome of the solution algorithm to the optimal solutions derived in the previous sections for randomly generated cases with separable mixing or two populations. For these problems our proposed solution algorithm is able to find the optimal solution within seconds.

Generating a random start solution that is feasible with respect to constraints (26) - (29) is not trivial, as the feasible region is not convex by Lemma 14. We propose the following approach to generate start solutions that satisfy most constraints. We can easily generate a random unit vector in $(0,1)$, that satisfies constraints $(27)$ - (28). We then determine $f_{i}$ with constraint (26) and set $f_{i}=0$ in case this results in a negative vaccination fraction. This guarantees (29) and possibly also constraints (26) for some $i \in J$. 


\section{Case study}

We apply our optimization model to a case study using the model and parameter values of Wallinga et al. (2010).

\subsection{Case description}

An age-structured population is considered with six age groups: 0-5, 6-12, 13-19, 20-39, 40-59 and 60+. The population sizes $N_{j}$ and contact parameters, denoted by $\delta_{i j}$ are presented respectively in Table 1 and Table 2.

\begin{tabular}{llllllll}
\hline Age group & $0-5$ & $6-12$ & $13-19$ & $20-39$ & $40-59$ & $60+$ & total \\
\hline Population $\left(\times 10^{3}\right)$ & 1060 & 1265 & 1642 & 4857 & 3312 & 2477 & 14613 \\
\hline
\end{tabular}

Table 1: The population sizes of the different age groups.

\begin{tabular}{cllllll}
\hline$\delta_{i j}\left(10^{-7}\right.$ day $\left.^{-1}\right)$ & $0-5$ & $6-12$ & $13-19$ & $20-39$ & $40-59$ & $60+$ \\
\hline $0-5$ & 1.393 & 0.259 & 0.146 & 0.284 & 0.130 & 0.094 \\
$6-12$ & 0.259 & 2.261 & 0.266 & 0.287 & 0.170 & 0.095 \\
$13-19$ & 0.146 & 0.266 & 1.847 & 0.418 & 0.309 & 0.123 \\
$20-39$ & 0.284 & 0.287 & 0.418 & 0.623 & 0.407 & 0.207 \\
$40-59$ & 0.130 & 0.170 & 0.309 & 0.407 & 0.504 & 0.272 \\
$60+$ & 0.094 & 0.095 & 0.123 & 0.207 & 0.272 & 0.447 \\
\hline
\end{tabular}

Table 2: The age-specific contact matrix.

The disease is modeled with a heterogeneous SIR model, see Section 2.1. The transmission rates $\beta_{i j}$ as presented in this paper can be calculated as follows: $\beta_{i j}=\delta_{i j} N_{j}$, with $\delta_{i j}$ being the proportion of age group $i$ contacted by an infected individual in age group $j$ per unit of time. The recovery rate is assumed to be the same for every age group: $\gamma_{j}=0.286$ for all $j \in J$. This results in an expected duration in the infected compartment (i.e., the generation interval) of 3.5. The reproduction ratio without vaccination is equal to $\rho(\mathbf{B} \gamma)=2.1$. The parameter values for both the generation interval and the reproduction ratio are in line with other studies in literature (Boëlle, Ansart, Cori, \& Valleron, 2011; Vink, Bootsma, \& Wallinga, 2014). 


\subsection{Solution}

We solve our optimization problems to determine the optimal vaccine allocation. This optimal allocation is compared to the following four allocation schemes:

- Random allocation - The vaccines are allocated at random (i.e., pro rata) over the age groups.

- Greedy allocation - The age groups are prioritized in accordance to the priority order presented in Algorithm 1.

- High-infection scheme 1 - The age groups are prioritized based on the final size as a fraction of the age group size, which results in the following order: 13-19, 20-39, 6-12, 40-59, 0-5, 60+.

- High-infection scheme 2 - The prioritization is determined based on the absolute final sizes (i.e., fractional final size weighted by age group size). This leads to the following priority order: 20-39, 40-59, 13-19, 60+, 6-12, 0-5.

A high-infection risk scheme is also studied by Mylius, Hagenaars, Lugnér, and Wallinga (2008). To determine the priority order in these allocation schemes we determine for every age group the expected final size (i.e., infection attack rate) without vaccination. These final sizes are presented in Figure 2.
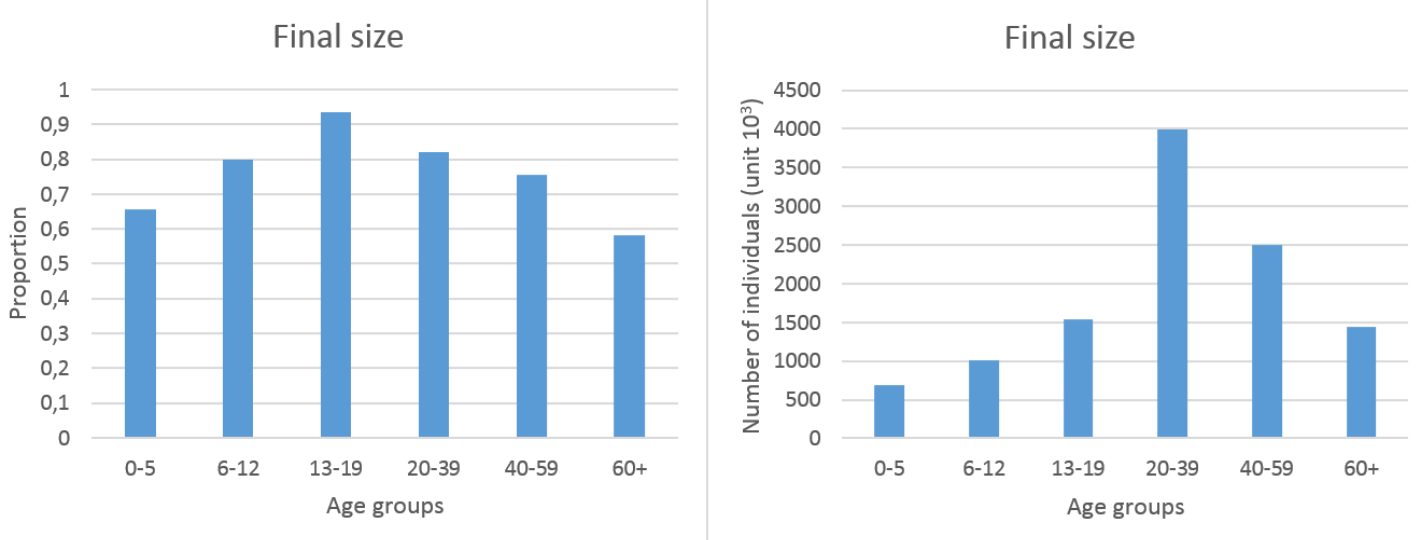

Figure 2: The final size without vaccination for the different age groups in fractions of the age group size (left) and in absolute numbers (right).

For the greedy allocation and the high-infection schemes we vaccinate according to the priority order until we achieve the threshold $R_{f}=1$ in a similar way as in Algorithm 1. The resulting vaccine allocations are presented in Table 3 .

We compare the vaccine allocation schemes in terms of the herd effect and the required vaccine stockpile to achieve the threshold $R_{f}=1$. The differences between the different allocations can be substantial, as can 


\begin{tabular}{lllllll}
\hline & \multicolumn{7}{c}{ Age groups } \\
& $0-5$ & $6-12$ & $13-19$ & $20-39$ & $40-59$ & $60+$ \\
\hline Optimal allocation & 0.000 & 0.515 & 0.862 & 0.791 & 0.000 & 0.000 \\
Random allocation & 0.530 & 0.530 & 0.530 & 0.530 & 0.530 & 0.530 \\
Greedy allocation & 1.000 & 1.000 & 1.000 & 0.635 & 0.000 & 0.000 \\
High-infection scheme 1 & 0.000 & 0.124 & 1.000 & 1.000 & 0.000 & 0.000 \\
High-infection scheme 2 & 0.000 & 0.022 & 1.000 & 1.000 & 1.000 & 1.000 \\
\hline
\end{tabular}

Table 3: The optimal vaccination fractions in every age group.

be seen in Table 4.

\begin{tabular}{lcc}
\hline & Herd effect & Required vaccine stockpile \\
\hline Optimal allocation & 8.705 & 5.908 \\
Random allocation & 6.867 & 7.746 \\
Greedy allocation & 7.563 & 7.050 \\
High-infection scheme 1 & 7.957 & 6.656 \\
High-infection scheme 2 & 2.297 & 12.316 \\
\hline
\end{tabular}

Table 4: The herd effect and required vaccine stockpile to attain $R_{f}=1$ for different allocation schemes (unit $\left.10^{6}\right)$.

The results in Table 4 show that the optimal allocation significantly outperforms the other allocation schemes. High-infection scheme 1 results in the second best performance, but nevertheless achieves a herd effect of approximately $9 \%$ below the optimum. High-infection scheme 2 results by far in the lowest herd effect (and equivalently in the largest required vaccine stockpile). Even when we disregard this worst performing allocation scheme, the optimal allocation still increases the herd effect with 9 to $26 \%$.

We analyzed the robustness of our optimal allocation for different values of $\gamma$. The analysis shows that the optimal allocation only changes minimally, for example for $\gamma=0.30$ the optimal allocation for the consecutive age groups is as follows: $\mathbf{f}=[0,0.4905,0.8545,0.7297,0,0]$. 


\section{Discussion}

In this paper we use the $S I R$ model with interacting populations to illustrate the derivation of the expression for the herd effect and the next generation matrix. We stress that our results will also hold for more general class of epidemic models. The basic reproduction ratio is derived as the largest eigenvalue of this next generation matrix. The herd effect function is a rewritten version of the final size formula of which the generality is shown in Ma and Earn (2006). We refer to Van den Driessche and Watmough (2002) and Diekmann et al. (2012) for the derivation of next generation matrices and basic reproduction ratios for general compartmental models. Van den Driessche and Watmough (2002) also shows that for these general models the disease free equilibrium is stable if $R_{0}<1$ and unstable if $R_{0}>1$. Thus, the results in this paper hold for more general compartmental models than the SIR model.

The results derived in the Case Study in Section 6 show that the optimal allocation significantly outperforms other allocations. To determine the optimal allocation we assume that the disease parameters $\mathbf{B}$ and $\gamma$ are known, which may be problematic in practice. In case there is no information about the parameters available, one could use observable data to estimate the disease parameters (cf. Wallinga, Teunis, \& Kretzschmar, 2006). We performed additional numerical analysis which indicates that the optimal allocation is robust to small changes in the parameter $\gamma( \pm 10 \%)$. Our methods could thus be used with the best parameter estimates. When even estimates of parameters are not available, simple allocation rules could be constructed that do not rely on parameters but only on observable information (cf. Wallinga et al., 2010).

In the formulation of the optimization problems in Section 2.4 we assume an unlimited supply of vaccines. For the problem of minimizing the required amount of vaccines under the constraint that $R_{f}=1$, this is needed to ensure that the constraint can be satisfied. The second optimization problem considers maximization of the herd effect. To prove equivalence of the two problems we also assumed unlimited supply of vaccines in the second problem. However, it would be interesting to study the allocation that maximizes the herd effect under a limited vaccine stockpile. This changes the problem for vaccine stockpiles that are insufficient to prevent an outbreak. Namely, in those cases vaccination cannot lead to $R_{f}=1$. In previous work we analyzed this problem of maximizing the herd effect under a capacity constraint on the vaccine stockpile for weakly interacting populations (Duijzer et al., 2015). Further research is needed to investigate the effects of adding a capacity constraint to the maximization of the herd effect for general types of interaction.

In Section 5.3 we present a solution approach for the general problem. To use Perron-Frobenius theory we assume that the matrix $\mathbf{F B} \gamma$ is irreducible. Although irreducibility is technically lost in case $f_{k}=1$ for some 
$k \in J$, this does not affect our solution method. In that case $R_{f}=\rho(\mathbf{F B} \gamma)$ is equal to the largest eigenvalue of the reduced matrix that contains only the rows and columns of $\mathbf{F B} \gamma$ that correspond to populations with a vaccination fraction strictly smaller than 1 (cf. Section 7.2 of Diekmann et al., 2012). This reduced matrix is nonnegative and irreducible.

In this paper we apply optimization techniques to determine vaccine allocations. The results from the case study in Section 6 show that the optimal allocation significantly outperforms other allocations. This study focuses on the entire time horizon of the epidemic and assumes that we are able to reduce the reproduction ratio to 1 . In case you are only interested in reducing the reproduction ratio on the short term, other allocations such as the ones proposed by Wallinga et al. (2010) might be better. Nevertheless, the differences in performance between the different allocations in our case study are such that it is worthwhile to investigate other application possibilities of our optimal allocation and optimization in general. For example, further analysis of the optimization results could be used to derive decision rules to construct vaccination policies that perform close to optimal.

\section{Conclusion}

In this paper we analyze the impact of vaccination on the reproduction ratio and the herd effect. A compartmental model for disease progression is used to model the outbreak. We prove that a vaccine allocation maximizes the overall herd effect if and only if $R_{f}=1$.

We formulate two optimization problems: finding a vaccine allocation that minimizes the number of vaccines needed to attain $R_{f}=1$ and finding a vaccine allocation that maximizes the herd effect. We show that these two problems are equivalent. Based on this equivalence result we propose solution methods. For two special cases the optimal solution can be characterized completely: For separable mixing we provide an exact greedy algorithm and for two populations we derive an explicit solution. For the general case an efficient solution approach is presented based on Perron-Frobenius theory. We illustrate this solution approach for a case study. The results show that using our optimization problem to derive vaccine allocations can increase the herd effect by 9 to $26 \%$. Equivalently, using our optimal allocation we are able to significantly reduce the required vaccine stockpile to attain $R_{f}=1$.

The optimal allocations derived in this paper potentially have wider applicability than for the prepandemic situation discussed in this paper. Further research is needed to investigate these application possibilities for optimization in vaccine allocation. 


\section{Acknowledgements}

The authors want to thank Prof. Odo Diekmann for his useful input. 


\section{Appendix A Analysis of the SIR model}

Lemma 15. For initial values $s_{j}(0), i_{j}(0), r_{j}(0) \in[0,1]$ and $s_{j}(0)+i_{j}(0)+r_{j}(0)=1$ for all $j \in J$, the solution $\left(s_{j}(t), i_{j}(t), r_{j}(t)\right)$ to (1) satisfies the following conditions:

(i) $s_{j}(t)+i_{j}(t)+r_{j}(t)=1$ for all $j \in J$ and at any time $t \geq 0$

(ii) $s_{j}(t), i_{j}(t), r_{j}(t) \in[0,1]$ for all $j \in J$ and at any time $t \geq 0$

(iii) $s_{j}(t)$ is non-increasing over time and $r_{j}(t)$ is non-decreasing over time for all $j \in J$

Proof. We prove the statements consecutively.

(i) Immediate from $\frac{d s_{j}}{d t}+\frac{d i_{j}}{d t}+\frac{d r_{j}}{d t}=0$.

(ii) By item (i) it suffices to prove that $s_{j}(t), i_{j}(t), r_{j}(t)$ are nonnegative for all $j \in J$. Note that the differential equations in (1) are continuous. Assume by contradiction that $s_{j}\left(t_{2}\right)<0$ at $t_{2}>0$. Due to the continuity of the differential equations there must be a time $0<t_{1}<t_{2}$ at which $s_{j}\left(t_{1}\right)=$

0 . However, by (1) we then have $\frac{d s_{j}}{d t}=0$, implying that $s_{j}(t)$ must then stay 0 . We arrive at a contradiction: it is not possible for $s_{j}(t)$ to become negative. Analogous we can prove that $i_{j}(t)$ is nonnegative, since $\frac{d i_{j}}{d t} \geq 0$ when $i_{j}\left(t_{3}\right)=0$ for some $t_{3}>0$. Finally, $i_{j}(t)$ being nonnegative implies that $r_{j}(t)$ is non-decreasing and thus $r_{j}(t)$ is also nonnegative. We proved the lemma for a single population $j$, but the proof applies to all $j \in J$.

(iii) The result follows directly from the differential equations in (1) and the nonnegativity of $s_{j}(t)$ and $i_{j}(t)$ for all $j \in J$ and for any time $t$ proven in item (ii).

Theorem 4. Given the initial values $s_{j}(0), i_{j}(0), r_{j}(0) \in[0,1]$ such that $s_{j}(0)+i_{j}(0)+r_{j}(0)=1$ for all $j \in J$ the differential equations in (1) have a unique solution at any time $t$.

Proof. We will also show that they are Lipschitz continuous: a function $f(\mathbf{x}): \mathbb{R}^{n} \rightarrow \mathbb{R}$ is Lipschitz continuous if and only if there is a bounded nonnegative constant $K$ and a norm such that:

$$
\left|f\left(\mathbf{x}_{1}\right)-f\left(\mathbf{x}_{2}\right)\right| \leq K|| \mathbf{x}_{1}-\mathbf{x}_{2}||
$$

We prove Lipschitz continuity for one population, but the proof applies to all populations $j \in J$. Denote by $\mathbf{x}=\left[s_{1}(t), \ldots, s_{n}(t), i_{1}(t), \ldots, i_{n}(t)\right]$ and define the functions $f_{1}(\mathbf{x})=\frac{d s_{j}(t)}{d t}, f_{2}(\mathbf{x})=\frac{d i_{j}(t)}{d t}, f_{3}(\mathbf{x})=\frac{d r_{j}(t)}{d t}$. By 
Lemma 15 we have $0 \leq \mathrm{x} \leq 1$ element wise. We derive the following, using the $\ell_{1}$-norm:

$$
\begin{aligned}
\left|f_{1}\left(\mathbf{x}_{\mathbf{1}}\right)-f_{1}\left(\mathbf{x}_{\mathbf{2}}\right)\right| & =\left|-\sum_{l \in J} \beta_{j l} s_{j}^{1} i_{l}^{1}+\sum_{l \in J} \beta_{j l} s_{j}^{2} i_{l}^{2}\right| \\
& =\left|\left(s_{j}^{2}-s_{j}^{1}\right) \sum_{l \in J} \beta_{j l} i_{l}^{1}+s_{j}^{2} \sum_{l \in J} \beta_{j l}\left(i_{l}^{2}-i_{l}^{1}\right)\right| \\
& \leq \sum_{l \in J} \beta_{j l}\left(\left|\left(s_{j}^{2}-s_{j}^{1}\right)\right|+\left|\sum_{l \in J}\left(i_{l}^{2}-i_{l}^{1}\right)\right|\right) \\
& \leq \sum_{l \in J} \beta_{j l}\left\|\mathbf{x}_{\mathbf{1}}-\mathbf{x}_{\mathbf{2}}\right\|_{1}
\end{aligned}
$$

For $f_{2}(\mathbf{x})$ we derive that:

$$
\begin{aligned}
\left|f_{2}\left(\mathbf{x}_{\mathbf{1}}\right)-f_{2}\left(\mathbf{x}_{\mathbf{2}}\right)\right| & =\left|\sum_{l \in J} \beta_{j l} s_{j}^{1} i_{l}^{1}-\gamma_{j} i_{j}^{1}-\sum_{l \in J} \beta_{j l} s_{j}^{2} i_{l}^{2}+\gamma_{j} i_{j}^{2}\right| \\
& =\left|\left(s_{j}^{1}-s_{j}^{2}\right) \sum_{l \in J} \beta_{j} i_{l}^{1}+s_{j}^{2} \sum_{l \in J: l \neq j} \beta_{j l}\left(i_{l}^{1}-i_{l}^{2}\right)+\left(s_{j}^{2} \beta_{j j}-\gamma_{j}\right)\left(i_{j}^{1}-i_{j}^{2}\right)\right| \\
& \leq\left(\gamma_{j}+\sum_{l \in J} \beta_{j l}\right)\left(\left|\left(s_{j}^{1}-s_{j}^{2}\right)\right|+\left|\sum_{l \in J: l \neq j}\left(i_{l}^{1}-i_{l}^{2}\right)\right|+\left|\left(i_{j}^{1}-i_{j}^{2}\right)\right|\right) \\
& \leq\left(\gamma_{j}+\sum_{l \in J} \beta_{j l}\right)\left\|\mathbf{x}_{\mathbf{1}}-\mathbf{x}_{\mathbf{2}}\right\|_{1}
\end{aligned}
$$

Finally, we also conclude that $f_{3}(\mathbf{x})$ is Lipschitz continuous:

$$
\left|f_{3}\left(\mathbf{x}_{1}\right)-f_{3}\left(\mathbf{x}_{2}\right)\right|=\left|\gamma_{j} i_{j}^{1}-\gamma_{j} i_{j}^{2}\right| \leq \gamma_{j}\left\|\mathbf{x}_{1}-\mathbf{x}_{\mathbf{2}}\right\|_{1}
$$

Given the initial value and the Lipschitz continuous differential equations, we can apply the Picard-Lindelöf Theorem (Lindelöf, 1894). This theorem states that there is a unique solution to the differential equations for any point in time, which completes the proof.

Lemma 5. For all $j \in J$ the following holds:

(i) $0 \leq G_{j}(\mathbf{f}) \leq \min \left\{\left(1-f_{j}\right), \frac{1}{\sigma_{j}}\right\}$

(ii) $G_{j}(\mathbf{f})=0$ if and only if $f_{j}=1$.

Proof. We can rewrite (3) using the Lambert W function (cf. Corless, Gonnet, Hare, Jeffrey, \& Knuth, 1996; Ma \& Earn, 2006):

$$
G_{j}(\mathbf{f})=\frac{-1}{\sigma_{j}} W\left[-\sigma_{j}\left(1-f_{j}\right) \exp \left\{-\sigma_{j}\left(\left(1-f_{j}\right)+\frac{1}{\sigma_{j}} \sum_{l \in J: l \neq j} \frac{\beta_{j, l}}{\gamma_{l}}\left[1-f_{l}-G_{l}(\mathbf{f})\right]\right)\right\}\right]
$$


Denote by $B_{j}(\mathbf{f})$ the following function, such that $G_{j}(\mathbf{f})=\frac{-1}{\sigma_{j}} W\left[B_{j}(\mathbf{f})\right]$ :

$$
B_{j}(\mathbf{f})=-\sigma_{j}\left(1-f_{j}\right) \exp \left\{-\sigma_{j}\left(\left(1-f_{j}\right)+\frac{1}{\sigma_{j}} \sum_{l \in J: l \neq j} \frac{\beta_{j l}}{\gamma_{l}}\left[1-f_{l}-G_{l}(\mathbf{f})\right]\right)\right\}
$$

Note that $\sigma_{j}>0$ for all $j \in J$ by Assumption 2. By definition of the Lambert $\mathrm{W}$ function $W(0)=0$ and $W(x) \in[-1,0)$ for $x \in\left[\frac{-1}{e}, 0\right)$. To complete the proof of this lemma, it therefore suffices to show that $B_{j}(\mathbf{f}) \in\left[\frac{-1}{e}, 0\right]$ and $B_{j}(\mathbf{f})=0$ if and only if $f_{j}=1$. From the nonnegativity of the exponential function, it follows directly that $B_{j}(\mathbf{f}) \leq 0$ and $B_{j}(\mathbf{f})=0$ if and only if $f_{j}=1$. Using that $G_{j}(\mathbf{f}) \leq\left(1-f_{j}\right)$ and that the function $f(y)=\frac{-y}{e^{y}}$ is minimized for $y=1$, the following holds:

$$
\begin{aligned}
B_{j}(\mathbf{f}) & =-\sigma_{j}\left(1-f_{j}\right) \exp \left\{-\sigma_{j}\left(\left(1-f_{j}\right)+\frac{1}{\sigma_{j}} \sum_{l \in J: l \neq j} \frac{\beta_{j l}}{\gamma_{l}}\left[1-f_{l}-G_{l}(\mathbf{f})\right]\right)\right\} \\
& \geq-\sigma_{j}\left(1-f_{j}\right) \exp \left\{-\sigma_{j}\left(1-f_{j}\right)\right\} \geq \frac{-1}{e}
\end{aligned}
$$

We showed that $0 \leq G_{j}(\mathbf{f}) \leq \frac{1}{\sigma_{j}}$ and that $G_{j}(\mathbf{f})=0$ if and only if $f_{j}=1$. To complete the proof of this lemma it suffices to show that $G_{j}(\mathbf{f}) \leq\left(1-f_{j}\right)$, which holds by Lemma 5 .

\section{Appendix B Theoretical results on $R_{f}$}

Lemma 16. The largest eigenvalue of a matrix $A$, denoted by $\rho(A)$, is a continuous function of $A$.

Proof. The eigenvalues of a matrix $A$ are equal to the roots of the characteristic polynomial of $A$. By Naulin and Pabst (1994) the roots of a polynomial depend continuously on the coefficients of the polynomial. That implies that the eigenvalues of $A$ are continuous in the matrix $A$. Let $A$ be a $n \times n$ matrix. Denote by a and b the vectors with elements $a_{i}$ and $b_{i}$ for $i=1, \ldots, n$, i.e., the vectors with the eigenvalues of the matrices $A$ and $B$ respectively. This implies that $\rho(A)=\max _{i}\left|a_{i}\right|$ and $\rho(B)=\max _{i}\left|b_{i}\right|$. For every $i$ the following holds:

$$
\begin{aligned}
\left|a_{i}\right| & \leq\left|b_{i}\right|+\left|a_{i}-b_{i}\right| \quad \text { by the triangle inequality } \\
& \leq \rho(B)+\|a-b\|_{\infty}
\end{aligned}
$$

This implies that $\rho(A) \leq \rho(B)+\|a-b\|_{\infty}$ and by symmetry also $\rho(B) \leq \rho(A)+\|a-b\|_{\infty}$. Thus, $|\rho(A)-\rho(B)| \leq$ $\|a-b\|_{\infty}$, meaning that the largest eigenvalue of a matrix continuously depends on the eigenvalues. Since the eigenvalues are continuously dependent on the matrix, the largest eigenvalue is a continuous function of the matrix. This completes the proof of this lemma. 
The largest eigenvalue of a matrix can be defined with Gelfand's formula, which holds for any norm:

$$
\rho(A)=\lim _{k \rightarrow+\infty}\left\|A^{k}\right\|^{\frac{1}{k}}
$$

Using Gelfand's formula we derive the following result:

Corollary 17. Let $A$ and $B$ be two nonnegative matrices with $A \leq B$ element wise. If the inequality between $A$ and $B$ is strict for at least one element, then $\rho(A)<\rho(B)$. Otherwise, $\rho(A) \leq \rho(B)$.

Proof. This proof is based on Meyer (2000) (Example 7.10.2). The Frobenius norm is defined as follows:

$$
\|A\|_{F}=\sqrt{\sum_{i} \sum_{j}\left|a_{i j}\right|^{2}}
$$

Note that the following holds:

$$
A \leq B \Rightarrow A^{k} \leq B^{k} \Rightarrow\left\|A^{k}\right\|_{F} \leq\left\|B^{k}\right\|_{F}
$$

The proof can easily be completed using (32):

$$
\left\|A^{k}\right\|_{F} \leq\left\|B^{k}\right\|_{F} \Rightarrow\left\|A^{k}\right\|_{F}^{\frac{1}{k}} \leq\left\|B^{k}\right\|_{F}^{\frac{1}{k}} \Rightarrow \lim _{k \rightarrow+\infty}\left\|A^{k}\right\|_{F}^{\frac{1}{k}} \leq \lim _{k \rightarrow+\infty}\left\|B^{k}\right\|_{F}^{\frac{1}{k}}
$$

Above result implies that $\rho(A) \leq \rho(B)$. Using the Frobenius norm we establish that $\left\|A^{k}\right\|_{F}<\left\|B^{k}\right\|_{F}$ when $A$ is strictly smaller than $B$ for at least one element. In that case we thus have $\rho(A)<\rho(B)$. This completes the proof.

From Corollary 17 we can derive the following result:

Lemma 18. Let $\mathbf{f}^{i}$ denote a vector of vaccination fractions with corresponding reproduction ratio $R_{f}\left(\mathbf{f}^{i}\right)$ for $i=1,2$. If $\mathbf{f}^{2} \geq \mathbf{f}^{1}$ for all elements and $\mathbf{f}^{1} \neq \mathbf{f}^{2}$, then $R_{f}\left(\mathbf{f}^{2}\right)<R_{f}\left(\mathbf{f}^{1}\right)$.

Proof. Denote by $\mathbf{F}_{1}$ and $\mathbf{F}_{2}$ the matrices with on the diagonal respectively the elements of $\left(1-\mathbf{f}^{1}\right)$ and $\left(1-\mathbf{f}^{2}\right)$. It holds that $R_{f}\left(\mathbf{f}^{2}\right)=\rho\left(\mathbf{F}_{\mathbf{2}} B \gamma\right)$ and $R_{f}\left(\mathbf{f}^{1}\right)=\rho\left(\mathbf{F}_{\mathbf{1}} B \gamma\right)$. We also have that $\mathbf{F}_{\mathbf{2}} \leq \mathbf{F}_{\mathbf{1}}$ with at least one element strict, because $\mathbf{f}^{1} \neq \mathbf{f}^{2}$. We can apply Corollary 17 :

$$
R_{f}\left(\mathbf{f}^{2}\right)=\rho\left(\mathbf{F}_{\mathbf{2}} B \gamma\right)<\rho\left(\mathbf{F}_{\mathbf{1}} B \gamma\right)=R_{f}\left(\mathbf{f}^{1}\right)
$$

Lemma 19. $R_{e}(t)$ is continuous in $t$. 
Proof. In Lemma 16 we show that the largest eigenvalue of a matrix $A$, denoted by $\rho(A)$, is a continuous function of $A$. From the differential equations (1) we conclude that $s_{j}(t)$ is a continuous function for all $j \in J$. By Definition 1 this implies that $R_{e}(t)$ is continuous in $t$, which completes the proof.

Corollary 20. $R_{e}(t)$ is monotonically non-increasing in $t$, i.e., $R_{e}(t+\epsilon) \leq R_{e}(t)$ for any $\delta>0$.

Proof. By Lemma $15 s_{j}(t)$ is non-increasing and thus $\mathbf{S}(\mathbf{t}+\delta) \leq \mathbf{S}(\mathbf{t})$. By Corollary 17 this implies: $R_{e}(t+$ $\delta)=\rho(\mathbf{S}(\mathbf{t}+\delta) \mathbf{B} \gamma) \leq \rho(\mathbf{S}(\mathbf{t}) \mathbf{B} \gamma)=R_{e}(t)$

Lemma 21. If $R_{f}>1$ then $\lim _{t \rightarrow+\infty} R_{e}(t)<1$. [Lemma 3.2 in Chan et al. (2014)]

\section{Appendix C Optimization Problem}

Lemma 13. For any solution to Problem (25) - (29) we have $f_{i} \geq 1-\frac{\gamma_{i}}{\beta_{i i}}$.

Proof. Let $k \in \arg \min _{j \in J \backslash\{i\}} \frac{\beta_{i j}}{\gamma_{j}}$. We derive the following relation for $f_{i}$ using (26) - (28):

$$
f_{i}=1-\frac{v_{i}}{\sum_{j \in J} \frac{\beta_{i j}}{\gamma_{j}} v_{j}} \geq 1-\frac{v_{i}}{\frac{\beta_{i k}}{\gamma_{k}}\left(1-v_{i}\right)+\frac{\beta_{i i}}{\gamma_{i}} v_{i}}
$$

To derive a lower bound on $f_{i}$ we maximize above expression with respect to $v_{i}$ :

$$
\frac{d}{d v_{i}}\left[1-\frac{v_{i}}{\frac{\beta_{i k}}{\gamma_{k}}\left(1-v_{i}\right)+\frac{\beta_{i i}}{\gamma_{i}} v_{i}}\right]=\frac{\frac{\beta_{i k}}{\gamma_{k}}}{\left[\frac{\beta_{i k}}{\gamma_{k}}\left(1-v_{i}\right) \frac{\beta_{i i}}{\gamma_{i}} v_{i}\right]^{2}} \geq 0
$$

By Assumption 2 the derivative is nonnegative and the expression is thus maximized for $v_{i}=1$. This proves the lower bound on $f_{i}: f_{i} \geq 1-\frac{\gamma_{i}}{\beta_{i i}}$.

Lemma 14. The feasible region of Problem (25) - (29) is not convex.

Proof. It suffices to give a counter example. Consider the case that $|J|=2$ and define the matrix $B \gamma$ and the variable vector $\mathbf{x}$ :

$$
B \gamma=\left[\begin{array}{ll}
1 & 3 \\
3 & 1
\end{array}\right] \quad \mathbf{x}\left[\left(1-f_{1}\right),\left(1-f_{2}\right), v_{1}, v_{2}\right]
$$

One can easily check that the following two vectors are feasible with respect to constraints (26) - (29): $\mathbf{x}_{\mathbf{1}}=\left[\begin{array}{llll}0.25 & 0.25 & 0.5 & 0.5\end{array}\right]$ and $\mathbf{x}_{\mathbf{2}}=\left[\begin{array}{llll}0.1 & 0.5 & 0.25 & 0.75\end{array}\right]$. Take the convex combination $\mathbf{x}_{\mathbf{3}}=\alpha \mathbf{x}_{\mathbf{1}}+(1-\alpha) \mathbf{x}_{\mathbf{2}}$

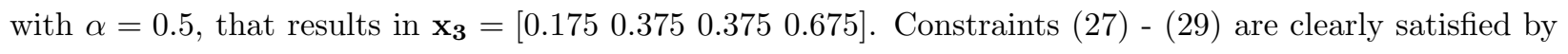
$\mathbf{x}_{\mathbf{3}}$. However, $\mathbf{x}_{\mathbf{3}}$ is not feasible with respect to constraint (26) nor to the relaxation in (30):

$$
0.175 \times[1 \times 0.375+3 \times 0.675] \approx 0.3937>0.375 \quad \text { and } \quad 0.375 \times[3 \times 0.375+1 \times 0.675] \approx 0.6563 \neq 0.675
$$


This completes the proof.

\section{Appendix D Vaccine efficacy}

In this paper we assumed that a vaccine is completely effective and leads to complete immunity. In literature this assumption is often relaxed by including a vaccine efficacy parameter (cf. Hill \& Longini Jr, 2003). In this section we study the effects of such a relaxation. Let $\psi_{j}$ denote the vaccine efficacy rate in population $j$. Vaccination then implies a shift from state $\left(s_{j}(0)=1, i_{j}(0)=0, r_{j}(0)=0\right)$ to state $\left(\left(1-\psi_{j} f_{j}\right), 0, \psi_{j} f_{j}\right)$ for all $j \in J$. Introducing a vaccine efficacy parameter is simply a rescaling of the vaccination fraction $f_{j}$. However, this rescaling has consequences for the equivalence of the two optimization problems defined in Section 4.2. As a result of reduced vaccine efficacy, the number of allocated vaccines is no longer equal to the number of effective vaccines. The total herd effect attained when $R_{f}=1$ is now equal to $\sum_{j \in J} N_{j}\left(1-\psi_{j} f_{j}\right)$,

whereas the number of allocated vaccines still equals $\sum_{j \in J} N_{j} f_{j}$. Thus, the last implication in the proof of Theorem 9 no longer holds. Only for the special case that $\psi_{j}=\psi$ for all $j \in J$ the equivalence still holds.

Without equivalence the two problems can still be solved with the solution approach of Section 5.3. The quadratic equality constraint in (26) can be reformulated as follows:

$$
\left(1-\psi_{i} f_{i}\right) \sum_{j \in J} \frac{\beta_{i j}}{\gamma_{j}} v_{j}=v_{i} \Leftrightarrow f_{i}=\frac{1}{\psi_{i}}\left[1-\frac{v_{i}}{\sum_{j \in J} \frac{\beta_{i j}}{\gamma_{j}} v_{j}}\right] \quad i \in J
$$

The objective function and the other constraints of Problem (25) - (29) remain valid for the problem of minimizing the required amount of vaccines to achieve $R_{f}=1$. For maximizing the herd effect the objective function must be changed to $\max \sum_{j \in J}\left(1-\psi_{j} f_{j}\right) N_{j}$.

\section{Appendix E Proof for $n=2$}

Theorem 12. The optimal solution to Problem (21) - (24) can be found among the boundary solutions given in Lemma 11 and the following solution:

$$
f_{1}=1-\frac{1}{d}\left[\sigma_{2}-\sqrt{\frac{N_{2}\left(\sigma_{1} \sigma_{2}-d\right)}{N_{1}}}\right] \quad \text { and } \quad f_{2}=1-\frac{1}{d}\left[\sigma_{1}-\sqrt{\frac{N_{1}\left(\sigma_{1} \sigma_{2}-d\right)}{N_{2}}}\right]
$$

For $d \leq 0$ only the boundary solutions need to be considered. 
Proof. By (20) we rewrite the objective function of Problem (21) - (24) into:

$$
O\left(f_{1}\right)=N_{1}\left(1-f_{1}\right)+N_{2}\left[\frac{1-\sigma_{1}\left(1-f_{1}\right)}{\sigma_{2}-\left(1-f_{1}\right) d}\right]
$$

We analyze the extrema of this function by setting the derivative of $O\left(f_{1}\right)$ with respect to $f_{1}$ equal to 0 :

$$
\begin{gathered}
\frac{d}{d f_{1}} O\left(f_{1}\right)=-N_{1}+\frac{N_{2}\left(\sigma_{1} \sigma_{2}-d\right)}{\left[\sigma_{2}-\left(1-f_{1}\right) d\right]^{2}}=0 \Leftrightarrow\left[\sigma_{2}-\left(1-f_{1}\right) d\right]^{2}=\frac{N_{2}\left(\sigma_{1} \sigma_{2}-d\right)}{N_{1}} \\
\Leftrightarrow f_{1}=1-\frac{1}{d}\left[\sigma_{2}-\sqrt{\frac{N_{2}\left(\sigma_{1} \sigma_{2}-d\right)}{N_{1}}}\right]
\end{gathered}
$$

In the third step we use (23). The objective function $O\left(f_{1}\right)$ thus has a unique extreme:

$$
f_{1}=1-\frac{1}{d}\left[\sigma_{2}-\sqrt{\frac{N_{2}\left(\sigma_{1} \sigma_{2}-d\right)}{N_{1}}}\right] \quad \text { and } \quad f_{2}=1-\frac{1}{d}\left[\sigma_{1}-\sqrt{\frac{N_{1}\left(\sigma_{1} \sigma_{2}-d\right)}{N_{2}}}\right]
$$

where $f_{2}$ is derived by substituting the expression for $f_{1}$ in (20). To verify whether this extreme is a minimum or a maximum, we analyze the second order derivative of $O\left(f_{1}\right)$ :

$$
\frac{d^{2}}{d\left(f_{1}\right)^{2}} O\left(f_{1}\right)=\frac{-2 d N_{2}\left(\sigma_{1} \sigma_{2}-d\right)}{\left[\sigma_{2}-\left(1-f_{1}\right) d\right]^{3}}
$$

Note that $\sigma_{1} \sigma_{2}-d=\frac{\beta_{12} \beta_{21}}{\gamma_{1} \gamma_{2}} \geq 0$. We distinguish between the following three cases: (a) $d=0$, (b) $d<0$ and (c) $d>0$. In case (a) the function $O\left(f_{1}\right)$ is linear. For case (b) the second order derivative is positive, implying that the extreme in (34) is a minimum. For both case (a) and (b) the function $O\left(f_{1}\right)$ is thus maximized in one of the boundary points. In case (c) the second order derivative is positive.

Thus, for $d \leq 0$ the optimal solution can be found among the two boundary solutions that are feasible according to Lemma 11. For $d>0$ the solution in (34) is a candidate for the optimal solution. However, this candidate possibly results in $f_{1}, f_{2}$ that violate constraints (23) or (24), rendering the candidate solution infeasible. Therefore, also the feasible boundary solutions must be compared. This completes the proof of this theorem. 


\section{References}

Andradóttir, S., Chiu, W., Goldsman, D., \& Lee, M. L. (2014). Simulation of influenza propagation: Model development, parameter estimation, and mitigation strategies. IIE Transactions on Healthcare Systems Engineering, 4(1), 27-48.

Andreasen, V. (2011). The final size of an epidemic and its relation to the basic reproduction number. Bulletin of Mathematical Biology, 73(10), 2305-2321.

Arino, J., Brauer, F., Van Den Driessche, P., Watmough, J., \& Wu, J. (2008). A model for influenza with vaccination and antiviral treatment. Journal of Theoretical Biology, 253(1), 118-130.

Ball, F. G., \& Lyne, O. D. (2002). Optimal vaccination policies for stochastic epidemics among a population of households. Mathematical Biosciences, 177, 333-354.

Becker, N. G., \& Starczak, D. N. (1997). Optimal vaccination strategies for a community of households. Mathematical Biosciences, 139(2), 117-132.

Boëlle, P.-Y., Ansart, S., Cori, A., \& Valleron, A.-J. (2011). Transmission parameters of the A/H1N1 (2009) influenza virus pandemic: a review. Influenza and Other Respiratory Viruses, 5(5), 306-316.

Britton, T., Becker, N. G., et al. (2000). Estimating the immunity coverage required to prevent epidemics in a community of households. Biostatistics, 1(4), 389-402.

Cairns, A. J. (1989). Epidemics in heterogeneous populations: aspects of optimal vaccination policies. Mathematical Medicine and Biology, 6(3), 137-159.

Chan, K. D. M., Nishiura, H., Diekmann, O., \& Bootsma, M. C. J. (2014). The impact of population ageing on the severity of an epidemic outbreak. (working manuscript)

Cooper, B. S., Pitman, R. J., Edmunds, W. J., \& Gay, N. J. (2006). Delaying the international spread of pandemic influenza. PLoS Medicine, 3(6), e212.

Corless, R. M., Gonnet, G. H., Hare, D. E., Jeffrey, D. J., \& Knuth, D. E. (1996). On the Lambert $W$ function. Advances in Computational Mathematics, 5(1), 329-359.

Dantzig, G. B. (1957). Discrete-variable extremum problems. Operations Research, 5(2), 266-288.

Diekmann, O. (1977). Limiting behaviour in an epidemic model. Nonlinear Analysis: Theory, Methods \& Applications, 1(5), 459-470.

Diekmann, O., Heesterbeek, H., \& Britton, T. (2012). Mathematical tools for understanding infectious disease dynamics. Princeton University Press.

Diekmann, O., \& Heesterbeek, J. (2000). Mathematical epidemiology of infectious diseases: model building, analysis and interpretation (Vol. 5). John Wiley \& Sons. 
Duijzer, L. E., van Jaarsveld, W. L., Wallinga, J., \& Dekker, R. (2015). Dose-optimal vaccine allocation over multiple populations (Tech. Rep.). Econometric Institute, Erasmus School of Economics. Retrieved from http://repub.eur.nl/pub/79212 (Report number: EI 2015-29)

Ferguson, N. M., Cummings, D. A., Cauchemez, S., Fraser, C., Riley, S., Meeyai, A., .. Burke, D. S. (2005). Strategies for containing an emerging influenza pandemic in Southeast Asia. Nature, 437(7056), 209214.

Goldstein, E., Apolloni, A., Lewis, B., Miller, J., Macauley, M., Eubank, S., ... Wallinga, J. (2009). Distribution of vaccine/antivirals and the least spread line in a stratified population. Journal of the Royal Society Interface, 7(46), 755-764.

Hethcote, H. W. (2000). The mathematics of infectious diseases. SIAM Review, 42(4), 599-653.

Hethcote, H. W., \& Van Ark, J. W. (1987). Epidemiological models for heterogeneous populations: proportionate mixing, parameter estimation, and immunization programs. Mathematical Biosciences, $84(1)$, $85-118$.

Hill, A. N., \& Longini Jr, I. M. (2003). The critical vaccination fraction for heterogeneous epidemic models. Mathematical Biosciences, 181(1), 85-106.

Hu, Z., Teng, Z., \& Jiang, H. (2012). Stability analysis in a class of discrete sirs epidemic models. Nonlinear Analysis: Real World Applications, 13(5), 2017-2033.

Keeling, M. J., \& Ross, J. V. (2015). Optimal prophylactic vaccination in segregated populations: When can we improve on the equalising strategy? Epidemics, 11, 7-13.

Keeling, M. J., \& Shattock, A. (2012). Optimal but unequitable prophylactic distribution of vaccine. Epidemics, 4(2), 78-85.

Lindelöf, E. (1894). Sur lapplication de la méthode des approximations successives aux équations différentielles ordinaires du premier ordre. Comptes rendus hebdomadaires des séances de lAcadémie des sciences, 114, 454-457.

Ma, J., \& Earn, D. J. (2006). Generality of the final size formula for an epidemic of a newly invading infectious disease. Bulletin of Mathematical Biology, 68(3), 679-702.

Metz, J. (1978). The epidemic in a closed population with all susceptibles equally vulnerable; some results for large susceptible populations and small initial infections. Acta biotheoretica, 27(1-2), 75-123.

Meyer, C. D. (2000). Matrix analysis and applied linear algebra. Siam.

Mylius, S. D., Hagenaars, T. J., Lugnér, A. K., \& Wallinga, J. (2008). Optimal allocation of pandemic influenza vaccine depends on age, risk and timing. Vaccine, 26(29), 3742-3749. 
Naulin, R., \& Pabst, C. (1994). The roots of a polynomial depend continuously on its coefficients. Rev. Colombiana Mat, 28, 35-37.

Ross, J. V., \& Black, A. J. (2014). Contact tracing and antiviral prophylaxis in the early stages of a pandemic: the probability of a major outbreak. Mathematical Medicine and Biology, dqu014.

Tildesley, M. J., \& Keeling, M. J. (2009). Is r0 a good predictor of final epidemic size: Foot-and-mouth disease in the uk. Journal of Theoretical Biology, 258(4), 623-629.

Van den Driessche, P., \& Watmough, J. (2002). Reproduction numbers and sub-threshold endemic equilibria for compartmental models of disease transmission. Mathematical Biosciences, 180(1), 29-48.

Vink, M. A., Bootsma, M. C. J., \& Wallinga, J. (2014). Serial intervals of respiratory infectious diseases: a systematic review and analysis. American Journal of Epidemiology, 180(9), 865-875.

Wallinga, J., \& Lipsitch, M. (2007). How generation intervals shape the relationship between growth rates and reproductive numbers. Proceedings of the Royal Society of London B: Biological Sciences, 274(1609), 599-604.

Wallinga, J., Teunis, P., \& Kretzschmar, M. (2006). Using data on social contacts to estimate age-specific transmission parameters for respiratory-spread infectious agents. American Journal of Epidemiology, $164(10), 936-944$.

Wallinga, J., van Boven, M., \& Lipsitch, M. (2010). Optimizing infectious disease interventions during an emerging epidemic. Proceedings of the National Academy of Sciences, 107(2), 923-928.

Waltz, R. A., Morales, J. L., Nocedal, J., \& Orban, D. (2006). An interior algorithm for nonlinear optimization that combines line search and trust region steps. Mathematical Programming, 107(3), 391-408.

Yuan, E. C., Alderson, D. L., Stromberg, S., \& Carlson, J. M. (2015). Optimal vaccination in a stochastic epidemic model of two non-interacting populations. PloS ONE, 10(2), e0115826. 